
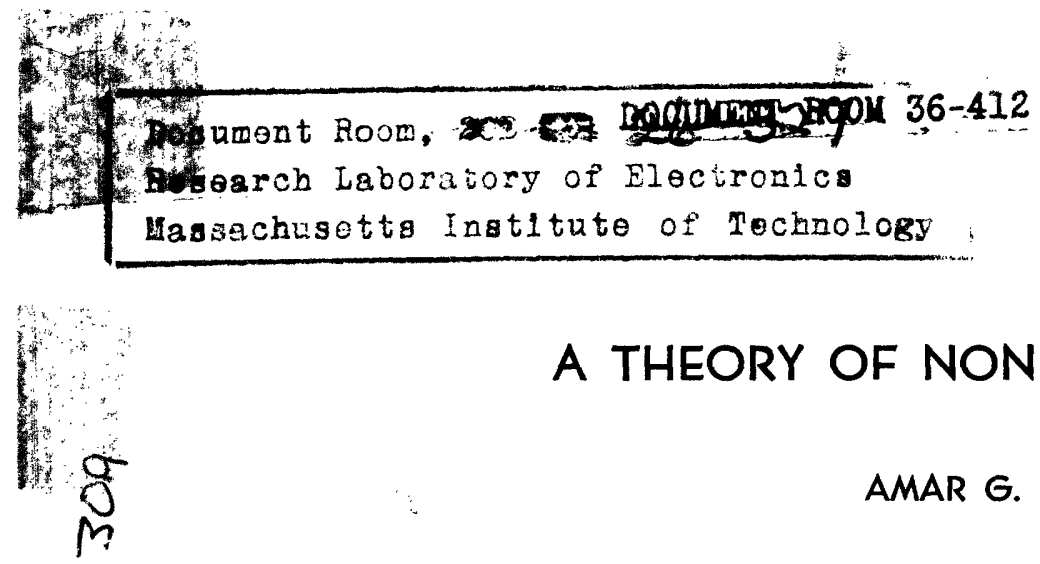

\title{
A THEORY OF NONLINEAR SYSTEMS
}

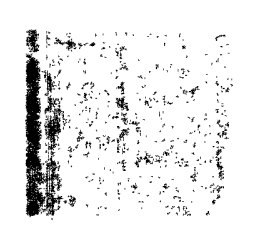

\section{LOAN.COPY-}

ONLY

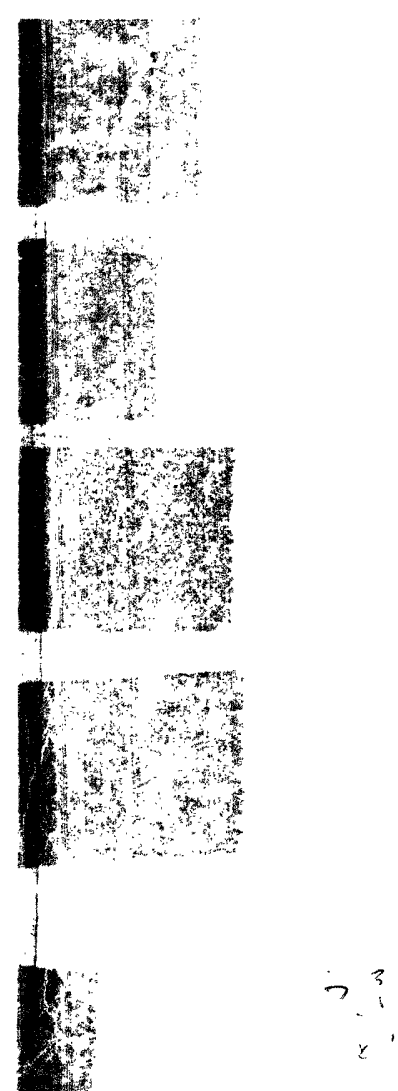

TECHNICAL REPORT 309

MAY 15, 1956 
The Research Laboratory of Electronics is an interdepartmental laboratory of the Department of Electrical Engineering and the Department of Physics.

The research reported in this document was made possible in part by support extended the Massachusetts Institute of Technology, Research Laboratory of Electronics, jointly by the U. S. Army (Signal Corps), the U. S. Navy (Office of Naval Research), and the U. S. Air Force (Office of Scientific Research, Air Research and Development Command), under Signal Corps Contract DA36-039-sc64637, Department of the Army Task No. 3-99-06-108 and Project No. 3-99-00-100. 


\title{
MASSACHUSETTS INSTITUTE OF TECHNOLOGY \\ RESEARCH LABORATORY OF ELECTRONICS
}

Technical Report 309

May 15, 1956

\section{A THEORY OF NONLINEAR SYSTEMS}

\author{
Amar G. Bose
}

This report is based on a thesis submitted to the Department of Electrical Engineering, M.I.T., May 14, 1956, in partial fulfillment of the requirements for the degree of Doctor of Science.

\begin{abstract}
The Wiener theory of nonlinear system characterization is described and some of its important concepts are discussed. Following these lines, a theory is developed for the experimental determination of optimum time-invariant nonlinear systems. The systems are optimum in a weighted mean-square sense in which the weighting function is at our disposal.

The design of nonlinear systems is regarded as the problem of mapping the function space of the past of the input onto a line that corresponds to the amplitude of the filter output. By choosing a series expansion for this mapping operation that partitions the function space into nonoverlapping cells, an orthogonal representation for nonlinear systems is obtained that leads to convenient apparatus for the determination of optimum systems. General methods are described for applying this theory to the determination of systems that have a performance superior to that of given linear or nonlinear systems. A criterion is established relative to which two systems are defined as "nearly equivalent" and the approximation of nonlinear systems by linear and simple nonlinear systems is discussed. The theory is extended to include the problem of multiple nonlinear prediction and apparatus for the determination of optimum predictors is indicated.
\end{abstract}




\section{INTRODUCTION}

A physically realizable nonlinear system, like a linear one, is a system whose present output is a function of the past of its input. We can regard the system as a computer that operates on the past of one time function to yield the present value of another time function. Mathematically, we say that the system performs a transformation on the past of its input to yield its present output. When this transformation is linear (the case of linear systems), we can take advantage of the familiar convolution integral to obtain the present output from the past of the input and the system is then said to be characterized by its response to an impulse. That is, the response of a linear system to an impulse is sufficient to determine its response to any input. When the transformation is nonlinear, we no longer have a simple relation like the convolution integral, which relates the output to the past of the input, and the system can no longer be characterized by its response to an impulse, since superposition does not apply. Wiener has shown, however, that we can characterize a nonlinear system by a set of coefficients and that these coefficients can be determined from a knowledge of the response of the system to shot-noise excitation. Thus, shot noise occupies the same position as a probe for investigating nonlinear systems that the impulse occupies as a probe for investigating linear systems. The first section of this report is devoted to the Wiener theory of nonlinear system characterization. Emphasis is placed on impor tant concepts of this theory that are used in succeeding sections to develop a theory for the determination of optimum nonlinear systems.

\section{THE WIENER THEORY OF NONLINEAR SYSTEM CHARACTERIZATION AND SYNTHESIS}

\subsection{GENERAL REMARKS}

The objectives of Wiener's method are: to obtain a set of coefficients which characterizes a time-invariant nonlinear system, and to present a procedure for synthesizing the system from a knowledge of its characterizing coefficients. An operator that relates the output to the past of the input of a nonlinear system is defined in such a way that the characterizing coefficients can be evaluated experimentally.

The method is confined to those nonlinear systems whose present behavior depends less and less upon the remote past of the input as we push this past back in time. More precisely, attention is restricted to those systems whose present output is influenced to an arbitrarily small extent by that portion of the past of the input beyond some arbitrarily large but finite time. Furthermore, we shall restrict our attention to those nonlinear systems that operate on continuous time functions to yield continuous time functions. This is clearly no physical restriction, since physical time functions, though they may change very rapidly, are continuous. The reasons for these restrictions will become apparent in the development of the theory that follows. 
According to Wiener, the most general probe for the investigation of nonlinear sys tems is gaussian noise with a flat power density spectrum, because there is a finite probability that this noise will, at some time, approximate any given time function arbitrarily closely over any finite time interval. Gaussian noise with a flat power density spectrum can be approximated by the output of a shot-noise generator. Hence, if two systems have the same response to shot noise, they will have the same response for any input, and we say that the systems are equivalent. The Wiener theory of nonlinear system classification is based on this property of the shot-noise probe. A given system is characterized by exciting it with shot noise and measuring certain averages of products of its output with functions of the shot-noise input which can be generated in the laboratory. The measured quantities are numerically equal to the coefficients in the Wiener nonlinear operator. Once these coefficients are determined, a system can be synthesized that yields the same response to shot noise as does the given system. Hence the two systems are equivalent.

Recognizing that the present output of a nonlinear system is a function of the past of its input, Wiener formulated his nonlinear operator by first characterizing the past of the time function on which it operates by a set of coefficients and then expressing the result of the operation (the system output) as an expansion of these coefficients (1). In the development that follows we shall treat these problems separately; first the problem of characterizing the past of a time function by a set of coefficients, and then the problem of expressing a nonlinear function of these coefficients.

\subsection{DEFINITIONS}

To simplify the description of the method, it is convenient at this point to define certain quantities and relations.

A. The $n^{\text {th }}$ Laguerre polynomial is defined (2) as

$$
L_{n}(x)=\frac{1}{(n-1) !} e^{x} \frac{d^{(n-1)}}{d x^{(n-1)}}\left(x^{(n-1)} e^{-x}\right) \quad n=1,2, \ldots
$$

B. The normalized Laguerre functions $h_{n}(x)$ are defined as

$$
h_{n}(x)= \begin{cases}e^{-x / 2} L_{n}(x) & x \geqslant 0 \\ 0 & x<0\end{cases}
$$

The following orthogonality relation exists for these functions:

$$
\int_{0}^{\infty} h_{m}(x) h_{n}(x) d x= \begin{cases}1 & \text { if } m=n \\ 0 & \text { if } m \neq n\end{cases}
$$


C. The $\mathrm{n}^{\text {th }}$ Hermite polynomial is defined (3) as

$$
F_{n}(x)=(-1)^{(n-1)} e^{x^{2}}\left(\frac{d}{d x}\right)^{(n-1)} e^{-x^{2}} \quad n=1,2,3, \ldots
$$

D. The normalized Hermite polynomials $\eta_{n}(x)$ are defined as

$$
\eta_{n}(x)=\frac{F_{n}(x)}{\left[2^{(n-1)}(n-1) !(\pi)^{1 / 2}\right]^{1 / 2}}
$$

E. The normalized Hermite functions are defined as

$$
\psi_{n}(x)=e^{-x^{2} / 2} \eta_{n}(x)
$$

These functions form a normal orthogonal set over the interval $-\infty$ to $\infty$. Consequently, we have the relation

$$
\int_{-\infty}^{\infty} \eta_{m}(x) \eta_{n}(x) e^{-x^{2}} d x= \begin{cases}1 & m=n \\ 0 & m \neq n\end{cases}
$$

\subsection{CHARACTERIZING THE PAST OF A TIME FUNCTION}

Given a time function $x(t)$, our object is to determine a set of coefficients that characterize its past. The coefficients are said to characterize the past of $x(t)$ if we can construct this past from a knowledge of them. Let us confine our attention here to real time functions $\mathrm{x}(\mathrm{t})$ that have the property

$$
\int_{-\infty}^{\infty} x^{2}(t) d t<\infty
$$

The past of such time functions can be expanded in a complete set of orthogonal functions. Furthermore, from a knowledge of the coefficients of this expansion, we can construct the time function almost everywhere (4). Because of their realization as the impulse response of rather simple networks, Wiener chose to expand the past of $x(t)$ in terms of Laguerre functions. These functions form a complete set over the interval 0 to $\infty$ and have the orthogonality property indicated in Eq. 2. The expansion of the past of $\mathrm{x}(\mathrm{t})$ in terms of the Laguerre functions is

$$
x(-t)=\sum_{n=1}^{\infty} u_{n} h_{n}(t) \quad t \geqslant 0
$$

where the present time is $t=0$ and the $u_{n}$ are the Laguerre coefficients of the past of $x(t)$. Taking advantage of the orthogonality property of Eq. 2, we obtain the following 


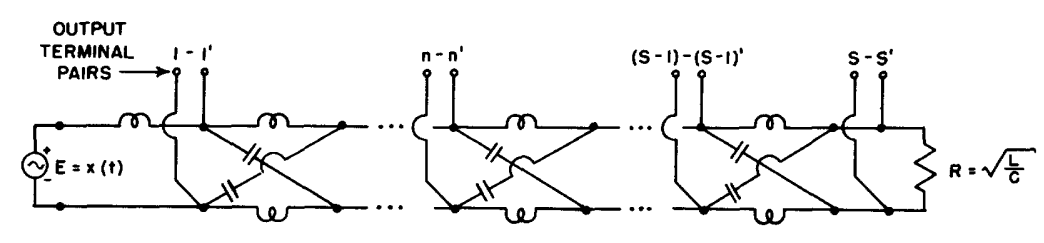

Fig. 1. Laguerre network. For $R=L=C=1$, the impulse response at the $\mathrm{n}^{\text {th }}$-output terminal pair is given by Eq. 1 .

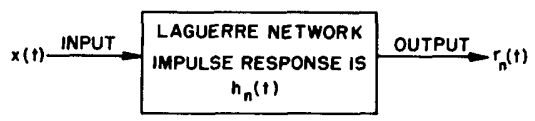

Fig. 2. Block diagram of Laguerre network showing $\mathrm{n}^{\text {th }}$ output.

expression for the $u_{n}$.

$$
u_{n}=\int_{0}^{\infty} x(-t) h_{n}(t) d t
$$

These Laguerre coefficients are readily generated, in practice, as the outputs of a rather simple network whose input is $x(t)$. This network, shown in Fig. 1 , is called a Laguerre network. It is a constant-impedance, lossless, ladder structure, terminated in its characteristic impedance, and preceded by a series inductance. For a detailed description of Laguerre networks, their analysis and synthesis, see reference 2. For our purposes, it is sufficient to know that the impulse response of the Laguerre network at the $n^{\text {th }}$-output terminal pair on open circuit is $h_{n}(t)$ for $n=1,2,3, \ldots$. We must now show that if $x(t)$ is applied to the input of this network, the output at the $n^{\text {th }}$ terminal pair at time $t=0$ is the $n^{\text {th }}$ Laguerre coefficient $u_{n}$ of the past of $x(t)$ up to the time $t=0$. To this end, we consider the block diagram of the Laguerre network shown in Fig. 2. For simplicity, only the $n^{\text {th }}$-output terminal is shown. The network input is $x(t)$. Its output $r_{n}(t)$ is given by the convolution of $x(t)$ with $h_{n}(t)$. That is,

$$
r_{n}(t)=\int_{0}^{\infty} x(t-\tau) h_{n}(\tau) d \tau
$$

At time $t=0$, the output is

$$
r_{n}(0)=\int_{0}^{\infty} x(-\tau) h_{n}(\tau) d \tau
$$

But the right side of this equation is seen to be equivalent to the expression for $u_{n}$ that is given in Eq. 7. Hence we see that if $x(t)$ is applied to the input of a Laguerre 
network, the output of the $n^{\text {th }}$ terminal pair at time $t=0$ is equal to the $n^{\text {th }}$ Laguerre coefficient of the past of $x(t)$ up to the time $t=0$. In general, the output of the $n^{\text {th }}$ terminal pair of the Laguerre network at any time $t$ is equal to the $n^{\text {th }}$ Laguerre coefficient of the past of the input up to the time $t$.

\subsection{PROPERTIES OF THE LAGUERRE COEFFICIENTS OF A SHOT-NOISE PROCESS}

Since the probe for the investigation of nonlinear systems in the Wiener theory is shot noise, it will be necessary in our development of this theory to make use of several properties of the Laguerre coefficients of a shot-noise process.

When the input of a Laguerre network is shot noise, the outputs (the Laguerre coefficients of the past of the shot-noise input) have the following three properties that are of interest:

1. They are gaussianly distributed.

2. They are statistically independent.

3. They all have the same variance.

The first property follows from the well-known result that the response of a linear system to a gaussian input is gaussian (5) (recall that shot noise is a gaussian time function with a flat power density spectrum).

The second property is proved as follows. Consider the Laguerre functions $h_{m}(t)$ and $h_{n}(t)$. Let $H_{m}(\omega)$ and $H_{n}(\omega)$ be the Fourier transforms of $h_{m}(t)$ and $h_{n}(t)$, respectively. Then $\mathrm{H}_{\mathrm{m}}(\omega)$ and $\mathrm{H}_{\mathrm{n}}(\omega)$ are the transfer functions from the input of the Laguerre network to the $m^{\text {th }}$ - and $n^{\text {th }}$-output terminal pairs. The cross-power density spectrum $\Phi_{n m}(\omega)$ of the $m^{\text {th }}$ - and $n^{\text {th }}$-output time functions can be expressed as

$$
\Phi_{n m}(\omega)=H_{m}(\omega) H_{n}^{* *}(\omega) \Phi_{i i}(\omega)
$$

where $\Phi_{i i}(\omega)$ is the input power density spectrum and the star denotes the complex conjugate of $H_{n}(\omega)(6)$. The crosscorrelation function $\phi_{n m}(\tau)$ of these output time functions is given by the Fourier transform of $\Phi_{n m}(\omega)$ as follows:

$$
\phi_{n m}(\tau)=\overline{f_{n}(t) f_{m}(t+\tau)}=\int_{-\infty}^{\infty} \Phi_{n m}(\omega) e^{j \omega \tau} d \omega
$$

in which the bar indicates averaging with respect to time. By the use of Eq. 9, Eq. 10 becomes

$$
\phi_{n m}(0)=\overline{f_{n}(t) f_{m}(t)}=\int_{-\infty}^{\infty} H_{m}(\omega) H_{n}^{*}(\omega) \Phi_{i i}(\omega) d \omega
$$

for $T=0$

If $\Phi_{\mathrm{ii}}(\omega)$ is a constant, then Eq. 11 can be written 


$$
\phi_{n m}(0)=\overline{f_{n}(t) f_{m}(t)}=\Phi_{i i}(\omega) \int_{-\infty}^{\infty} H_{m}(\omega) H_{n}^{*}(\omega) d \omega
$$

We now make use of the Parseval theorem to express the integral in Eq. 12 as follows:

$$
\frac{1}{2 \pi} \int_{-\infty}^{\infty} H_{m}(\omega) H_{n}^{*}(\omega) d \omega=\int_{0}^{\infty} h_{n}(t) h_{m}(t) d t
$$

Using the orthogonality property of the Laguerre functions (Eq. 2) in Eqs. 13 and 12, we obtain

$$
\overline{f_{n}(t) f_{m}(t)}= \begin{cases}2 \pi \Phi_{i i}(\omega) & m=n \\ 0 & m \neq n\end{cases}
$$

when $\Phi_{i i}(\omega)$ is a constant. Note that if $\Phi_{i i}(\omega)$ is a constant, it can have no impulse at the origin and thus the input and output time functions of the Laguerre network must have zero means. Therefore, we have shown that the outputs of the Laguerre network are linearly independent when the power density spectrum of the input is flat. (Note that this is true whether or not the input time function is gaussian and that it also holds for any orthogonal set of networks, not only for the Laguerre network.)

In the case of a shot-noise input, the Laguerre coefficients are gaussian time functions (property 1), and linear independence implies statistical independence; thus property 2 is proved.

Property 3 can be proved by solving for the variance of the $\mathrm{n}^{\text {th }}$ Laguerre coefficient in terms of the power density spectrum of the $n^{\text {th }}$ output of the network. However, it can be seen very simply by recalling that the Laguerre network, except for its first series inductance, is a constant-resistance, lossless structure terminated in its characteristic resistance. If, in Fig. 1, we look to the right at any of the output terminal pairs $n-n$, we see the characteristic resistance of the network. Since the structure is lossless, the same power flows through each section and, since the impedance at each section is resistive and the same for each section, the mean-square value of every Laguerre coefficient is the same. For a shot-noise input, the mean value of each coefficient is zero. Hence the variance $\sigma_{n}^{2}=\bar{u}_{n}^{2}(t)-{\overline{u_{n}(t)}}^{2}$ is the same for all Laguerre coefficients. In particular, if the level of the shot-noise input to the network of Fig. 1 is properly adjusted, all the Laguerre coefficients will have $\sigma^{2}=1$. In the development of the Wiener theory that follows we shall assume this to be the case.

In section 1.3 we restricted our attention to time functions that are square integrable over the interval $-\infty$ to $\infty$. In the present section we speak of applying shot noise to the input of the Laguerre network. This is justified by the fact that the past of any physical time function which we can generate as an input to our Laguerre network is square integrable, since it starts at some time in the finite past. 
Any practical application of the Wiener theory must, of course, use only a finite number of Laguerre coefficients to characterize the past of the system input. Since all the Laguerre functions decay exponentially (Eq. 1), for any finite number of these functions there exists some time in the finite past such that the present outputs of the Laguerre network are influenced to an arbitrarily small extent by the behavior of the input prior to this time. That is, for all practical purposes, the outputs of the Laguerre network are not cognizant of the past of the input beyond some finite time. Hence, as we mentioned in section 1.1, the application of the Wiener theory is restricted to systems whose present output is influenced to an arbitrarily small extent by that portion of the past of the input beyond some arbitrarily large but finite time.

\subsection{WIENER NONLINEAR OPERATOR}

Since the Laguerre coefficients characterize the past of a time function, any quantity dependent only on the past of this time function can be expressed as a function of these coefficients. Thus, for the nonlinear system with input $x(t)$ and output $y(t)$, we can write

$$
y(t)=F\left[u_{1}, u_{2}, \ldots, u_{s}, \ldots\right]
$$

in which the $u^{\prime}$ 's are the Laguerre coefficients of $x(t)$ at time $t$.

To put Eq. 15 in a more useful form, we must choose an expansion for the function $F$ of the Laguerre coefficients. These coefficients can take on any real value from $-\infty$ to $\infty$. The Hermite functions are chosen for the expansion because they form a complete orthonormal set over the interval $-\infty$ to $\infty$ and, as we shall see, are particularly adapted to a gaussian distribution. The expansion of Eq. 15 in terms of normalized Hermite functions, which are defined in Eq. 4, reads

$y(t)=\lim _{s \rightarrow \infty} \sum_{i=1}^{\infty} \sum_{j=1}^{\infty} \ldots \sum_{h=1}^{\infty} a_{i, j}, \ldots, h \eta_{i}\left(u_{1}\right) \eta_{j}\left(u_{2}\right) \ldots \eta_{h}\left(u_{s}\right) \exp \left(-\frac{u_{1}^{2}+u_{2}^{2}+\ldots+u_{s}^{2}}{2}\right)$

This equation expresses the amplitude of the time function $y(t)$ as a function of the Laguerre coefficients of the past of the time function $x(t)$. It can be simplified by letting $V(a)$ represent the product of polynomials $\eta_{i}\left(u_{1}\right) \eta_{j}\left(u_{2}\right) \ldots \eta_{h}\left(u_{s}\right)$ and $A_{a}$ represent the corresponding coefficient $a_{i, j}, \ldots, h$. Then Eq. 16 becomes

$$
y(t)=\lim _{s \rightarrow \infty} \sum_{a} A_{a} V(a) \exp \left(-\frac{u_{1}^{2}+u_{2}^{2}+\ldots+u_{s}^{2}}{2}\right)
$$

The behavior of any system of the class of systems considered in the Wiener theory can be expressed in the form of Eq. 17. The coefficients $A_{a}$ are said to characterize the system because the complete expression relating the output of the system $y(t)$ to the past 
of its input $\mathrm{x}(\mathrm{t})$, for any input time function, is known when the $\mathrm{A}_{a}$ are known.

We now come to the problem of characterizing a given nonlinear system, that is, the problem of evaluating the $\mathrm{A}_{\alpha}$ that correspond to a given nonlinear system. The object is to obtain an expression for the $\mathrm{A}_{a}$ suitable for experimental evaluation. To obtain such an expression, Wiener multiplies both sides of Eq. 17 by V $(\beta)$ and then makes use of the gaussian distribution of the Laguerre coefficients of a shot-noise process to obtain Eq. 26 for the $\mathrm{A}_{a}$. However, we shall use a different approach in order to arrive at Eq. 26 that will give us a better physical understanding of the Wiener method.

In the practical case, we shall always use a finite number of Laguerre coefficients and Hermite functions. Then the sum on the right side of Eq. 17 does not yield y(t) exactly but only approximates it. We can regard the finite sum

$$
\sum_{a} A_{a} V(a) \exp \left(-\frac{u_{1}^{2}+\ldots+u_{s}^{2}}{2}\right)
$$

as representing the output of a nonlinear system in terms of $s$ Laguerre coefficients of its input and a finite number of Hermite functions. We want to choose the $\mathrm{A}_{a}$ so that this sum best approximates the output $y(t)$ of the given system with respect to some error criterion when both systems have the same input. Since, according to section 1.1, the most general time function is shot noise, we shall let it be the common input. We define

$$
\mathscr{E}=\lim _{\mathrm{T} \rightarrow \infty} \frac{1}{2 \mathrm{~T}} \int_{-\mathrm{T}}^{\mathrm{T}} \exp \left(\frac{\mathrm{u}_{1}^{2}+\ldots+\mathrm{u}_{\mathrm{s}}^{2}}{2}\right)\left[\mathrm{y}(\mathrm{t})-\sum_{a} \mathrm{~A}_{a} \mathrm{~V}(a) \exp \left(-\frac{\mathrm{u}_{1}^{2}+\ldots+\mathrm{u}_{\mathrm{s}}^{2}}{2}\right)\right]^{2} \mathrm{dt}
$$

as the error between the outputs of the two systems. The justification of the choice of this weighted mean square error is that it leads to convenient independent expressions for the $\mathrm{A}_{\alpha}$, as we shall see. We now minimize $\mathscr{E}$ with respect to the $\mathrm{A}_{\alpha}$. In particular, for the coefficient $A_{\beta}$, we have

$\frac{\partial \mathscr{E}}{\partial \mathrm{A}_{\beta}}=\lim _{\mathrm{T} \rightarrow \infty} \frac{1}{2 \mathrm{~T}} \int_{-\mathrm{T}}^{\mathrm{T}}-2 \mathrm{~V}(\beta)\left[\mathrm{y}(\mathrm{t})-\sum_{a} \mathrm{~A}_{\alpha} \mathrm{V}(a) \exp \left(-\frac{\mathrm{u}_{1}^{2}+\ldots+\mathrm{u}_{s}^{2}}{2}\right)\right] \mathrm{dt}$

For the error to be a minimum with respect to $A_{\beta}$, we must set Eq. 20 to zero. This gives

$\lim _{T \rightarrow \infty} \frac{1}{2 T} \int_{-T}^{T} y(t) V(\beta) d t=\lim _{T \rightarrow \infty} \frac{1}{2 T} \int_{-T}^{T} V(\beta) \sum_{a} A_{a} V(a) \exp \left(-\frac{u_{1}^{2}+\ldots+u_{S}^{2}}{2}\right) d t$ 
We have seen (sec. 1.4) that the Laguerre coefficients of the past of a shot-noise process are statistically independent, normalized, gaussian variables. Thus the joint distribution of the Laguerre coefficients is

$$
P\left(u_{1}, \ldots, u_{s}\right)=(2 \pi)^{-s / 2} \exp \left(-\frac{u_{1}^{2}+\ldots+u_{s}^{2}}{2}\right)
$$

Our knowledge of this distribution is helpful in evaluating the integral on the right side of Eq. 21. Taking advantage of the ergodic theorem, we can replace the time average of the right side of Eq. 21 by the corresponding ensemble average, with the result:

$$
\begin{aligned}
\lim _{\mathrm{T} \rightarrow \infty} \frac{1}{2 \mathrm{~T}} \int_{-\mathrm{T}}^{\mathrm{T}} \mathrm{y}(\mathrm{t}) \mathrm{V}(\beta) \mathrm{dt}= & \int_{-\infty}^{\infty} \cdots \int_{-\infty}^{\infty} \mathrm{V}(\beta) \sum_{a} \mathrm{~A}_{a} \mathrm{~V}(a) \exp \left(-\frac{\mathrm{u}_{1}^{2}+\ldots+\mathrm{u}_{\mathrm{S}}^{2}}{2}\right) \\
& \times \mathrm{P}\left(\mathrm{u}_{1}, \ldots, \mathrm{u}_{\mathrm{S}}\right) \mathrm{du_{1 }} \ldots \mathrm{du_{ \textrm {s } }}
\end{aligned}
$$

After using Eq. 22 in Eq. 23 and interchanging the order of integration and summation, we obtain

$(2 \pi)^{s / 2} \overline{y(t) V(\beta)}=\sum_{a} A_{\alpha} \int_{-\infty}^{\infty} \ldots \int_{-\infty}^{\infty} V(a) V(\beta) \exp \left(-u_{1}^{2}+\ldots+u_{s}^{2}\right) d u_{1} \ldots d u_{s}$

in which the bar above $y(t) V(\beta)$ indicates the time average of this quantity. Since $V(a)$ and $V(\beta)$ are products of Hermite polynomials of the Laguerre coefficients, we can separate Eq. 24 into a product of integrals each of which involves only one Laguerre coefficient, as in the following equation:

$$
\begin{aligned}
& (2 \pi)^{s / 2} \overline{y(t) v(\beta)} \\
& \quad=\sum_{a} A_{a} \int_{-\infty}^{\infty} \eta_{i}\left(u_{1}\right) \eta_{i^{\prime}}\left(u_{1}\right) e^{-u_{1}^{2}} d u_{1} \ldots \int_{-\infty}^{\infty} \eta_{h^{\prime}}\left(u_{s}\right) \eta_{h^{\prime}}\left(u_{s}\right) e^{-u_{s}^{2}} d u_{s}
\end{aligned}
$$

In this equation the unprimed subscript indices belong to those Hermite polynomials that make up $\mathrm{V}(a)$, while the primed indices belong to those Hermite polynomials that make up $\mathrm{V}(\beta)$. If we recall the orthogonality property of the Hermite functions (Eq. 5), it is clear that unless all the primed indices $i^{\prime}, j^{\prime}, \ldots, h^{\prime}$ in Eq. 25 are equal to the corresponding unprimed indices $i, j, \ldots, h$, in other words, unless $\beta$ equals $a$, at least one of the integrals will be zero. By the same token, if $\beta=a$, then all the integrals have the value unity. Hence Eq. 25 reduces to 


$$
(2 \pi)^{\mathrm{s} / 2} \overline{\mathrm{y(t)} V(\beta)}=A_{\beta}
$$

which provides the basis for the experimental determination of the characterizing coefficients $A_{\alpha}$. The reason for the choice of Hermite functions to expand the right side of Eq. 15 now becomes apparent. The joint gaussian probability density of the Laguerre coefficients of the shot-noise input (Eq. 22) supplies the necessary exponential weighting factor in Eq. 23, thus enabling us to take advantage of the orthogonality of the Hermite functions in evaluating the coefficients $\mathrm{A}_{a}$.

This approach to the Wiener theory clearly points out that, for any given number of Laguerre coefficients and Hermite functions, this theory determines that system whose output best approximates (in the weighted mean-square sense of Eq. 19) the output of the given system for shot-noise input of both systems. As the number of Laguerre coefficients and Hermite functions is increased, the output (for shot-noise input) of any system of the Wiener class of systems can be approximated with vanishing error. And, from the discussion of section 1.1, if two systems have the same response to shot noise, then they have the same response to any common input and can be considered equivalent.

\subsection{EXPERIMENTAL APPARATUS FOR CHARACTERIZING AND SYNTHESIZING NONLINEAR SYSTEMS}

Equation 26 provides the basis for the experimental determination of the characterizing coefficients $A_{a}$. The apparatus for the determination of the coefficients $A_{a}$ is shown in Fig. 3. The output of a shot-noise generator is fed simultaneously into the given nonlinear system and into the Laguerre network. The output of the given nonlinear system is $y(t)$. The outputs of the Laguerre network are fed into a device involving multipliers and adders. This device generates products of Hermite polynomials (the V's) whose arguments are the Laguerre coefficients. Each output of this Hermite polynomial generator, when multiplied by $y(t)$ and averaged, yields, by Eq. 26, one of the characterizing coefficients of the given nonlinear system.

Having described the method for determining the characterizing coefficients of a nonlinear system, we now turn our attention to the Wiener method of synthesis of nonlinear systems from their characterizing coefficients. The general representation of

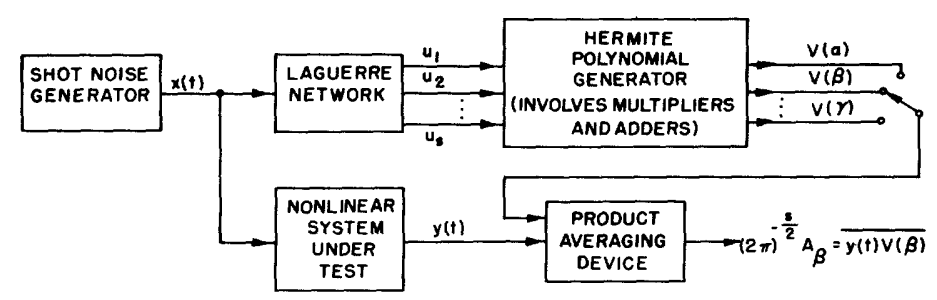

Fig. 3. Block diagram of the circuitry for the characterization of nonlinear systems. 


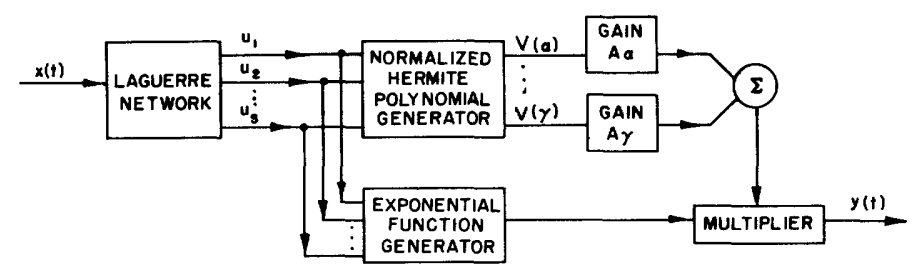

Fig. 4. Block diagram of the circuitry for the synthesis of nonlinear systems.

a nonlinear system is given by Eq. 17, which is the guide for the synthesis problem. This equation tells us that, for each $a$, we must generate $\mathrm{V}(a)$ and multiply it by $\mathrm{A}_{a}$ and $\exp \left[-\left(u_{1}^{2}+\ldots+u_{s}^{2}\right) / 2\right]$. Then each product must be added to give the system out put $y(t)$. In practice, the number of multipliers is reduced if we first form the sum of the products $\mathrm{A}_{\alpha} \mathrm{V}(a)$ and then multiply by the exponential function.

The exponential function, $\exp \left[-\left(u_{1}^{2}+\ldots+u_{s}^{2}\right) / 2\right]$, can be obtained as the product of $\mathbf{s}$ exponential function generators whose inputs are $u_{1}$ through $u_{s}$. Such generators give an output of $\exp \left(-u^{2} / 2\right)$ when the input is $u$. They are realizable, among other ways, in the form of a small cathode-ray tube with a special target which generates the $\exp \left(-\mathrm{u}^{2} / 2\right)$ function.

The block diagram of the apparatus for the synthesis procedure is shown in Fig. 4 .

EXAMPLE 1. In order to fix ideas, let us consider a simple example which is particularly suited to characterization and synthesis by the Wiener method. It should be emphasized that the Wiener method is an experimental method and that, for the purpose of illustrating mathematically how the method works, only the simplest of examples can be handled analytically. Let the example be a nonlinear system that contains no storage elements. Furthermore, let its output-input characteristic (transfer characteristic) be given by the equation

$$
y(t)=\exp \left(-x^{2}(t) / 2\right)
$$

In both the characterization and synthesis procedures that have been described, the function of the Laguerre network is to introduce dependence of the system output on the past of its input. The nonlinearity is brought about by the Hermite polynomial generator. For this simple example, there is no dependence upon the past and thus we can by-pass the Laguerre network. In the experimental procedure, the fact that this given nonlinear system has no storage could be determined from the results of a priori tests made on the system.

We notice that, as a result of by-passing the Laguerre network, the variables $u_{1}$ through $u_{s}$ (Fig. 3) are replaced by the single variable $x(t)$, as shown in Fig. 5. Equation 16 then becomes:

$$
y(t)=\sum_{i} a_{i} \eta_{i}(x) \exp \left(-x^{2} / 2\right)
$$




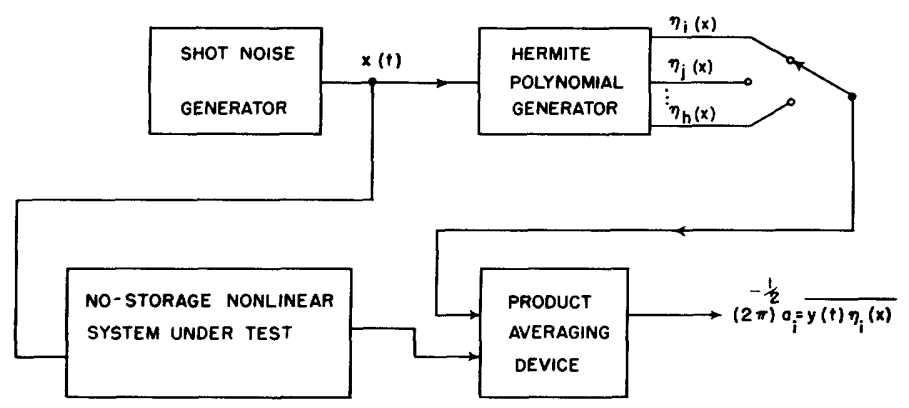

Fig. 5. Block diagram of the circuitry for the characterization of no-storage nonlinear systems.

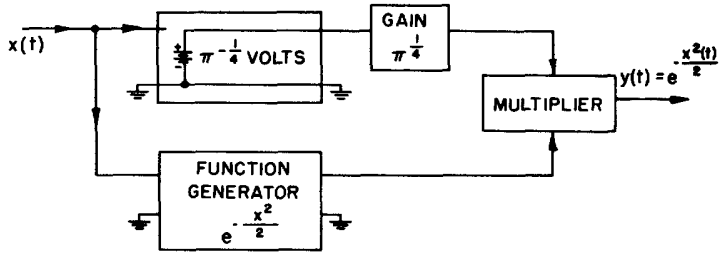

(a)

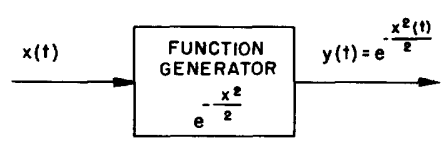

(b)

Fig. 6. (a) Synthesis of the nonlinear characteristic $y=\exp \left(-x^{2} / 2\right)$ in accordance with the general procedure of Fig. 4. (b) Equivalent reduced form of part (a).

and Eq. 26 becomes:

$$
a_{i}=(2 \pi)^{1 / 2} \overline{y(t) \eta_{i}(x)}
$$

Let us make use of the ergodic theorem to evaluate this time average as an ensemble average. Using Eq. 27, we can write

$$
a_{i}=(2 \pi)^{1 / 2} \int_{-\infty}^{\infty} \eta_{i}(x) \exp \left(-x^{2} / 2\right) P(x) d x
$$

But since, in the test circuits (Fig. 5), $x(t)$ is the output of an ideal shot-effect generator, the probability density of $\mathrm{x}$ is

$$
P(x)=(2 \pi)^{-1 / 2} \exp \left(-x^{2} / 2\right)
$$

Thus 


$$
a_{i}=\int_{-\infty}^{\infty} \eta_{i}(x) e^{-x^{2}} d x
$$

Referring to Eq. 3 and the definition of the Hermite polynomial, we see that

$$
\eta_{1}(x)=\pi^{-1 / 4}
$$

With this result, Eq. 32 can be written

$$
a_{i}=\pi^{1 / 4} \int_{-\infty}^{\infty} \eta_{i}(x) \eta_{1}(x) e^{-x^{2}} d x
$$

As a consequence of the orthogonality of the Hermite functions (Eq. 5), we have

$$
\begin{aligned}
& a_{1}=\pi^{1 / 4} \\
& a_{i}=0 \quad i \neq 1
\end{aligned}
$$

These coefficients serve to characterize the simple nonlinear system of this example.

Now let us synthesize the system from these coefficients. The guide for the synthesis is Eq. 28, which corresponds to Eq. 17 for the more complicated case involving storage. Since, from Eq. 35, only one coefficient is different from zero, the sum in Eq. 28 has only one term and can be written

$$
y(t)=a_{1} \eta_{1}(x) \exp \left(-x^{2} / 2\right)
$$

The synthesis of the system amounts to generating $\eta_{1}(x)$ and $\exp \left(-x^{2} / 2\right)$ and forming the product indicated in Eq. 36. The formal synthesis of the system, in accordance with the block diagram of Fig. 4, is shown in Fig. 6a. Since $\eta_{1}(x)$ is just a constant, independent of $x$, the system is seen to be equivalent to that of Fig. $6 \mathrm{~b}$. We see that, for example 1 , the synthesized system consists solely of the "function generator," a component which in the more complicated case will form only a part of the synthesized system.

\subsection{OBSERVATIONS AND COMMENTS}

It can be seen from Eq. 16 that if we choose to represent the past of the system input by $s$ Laguerre coefficients and if, furthermore, we decide to let the Hermite polynomial indices, $i, j, \ldots, h$ (Eq. 16), range from 1 to $n$, we have $n^{s}$ coefficients $A_{a}$ to evaluate. Without a doubt this number can become quite large in many cases of practical interest. However, with the freedom that exists in nonlinear systems we can hardly expect to apply such a general approach without a great deal of effort. At present, the large number of multipliers that is required for the generation of the Hermite polynomials and their products is the principal deterrent to the practical application 
of the Wiener method of characterization and synthesis. It is safe to say that, at present, the Wiener theory is of greater theoretical than practical interest.

One of the most significant contributions of the Wiener theory is that it shows us that any nonlinear system, of the broad class of systems considered by this theory, can be synthesized as a linear network with multiple outputs cascaded with a nonlinear circuit that has no memory of the past (Fig. 4). The linear network (the Laguerre network) serves to characterize the past of the input and the nonlinear no-storage circuit performs a nonlinear operation on the present outputs of the linear network to yield the system output. Thus, regardless of how the linear and nonlinear operations occur in any given circuit, the same over-all operation can be achieved by a linear operation followed by a nonlinear one, as shown in Fig. 4.

Another important contribution of the Wiener theory is the concept of the shot-noise probe for a nonlinear system. Just as the response of a linear system to an impulse is sufficient to characterize the system, so Wiener has shown that the response of a nonlinear system to shot noise is sufficient to characterize it.

In the Wiener theory two parameters remain free: the time-scale factor of the Laguerre functions and the scale factor in the argument of the Hermite functions. For convenience, both have been taken as unity in the preceding development. We may choose these as we desire in order to reduce the apparatus that is necessary for performing a given operation. Unfortunately, we have no simple procedure for determining the optimum values of these scale factors to enable us to do the best job with a given number of Laguerre coefficients and Hermite functions. We shall see a possible approach to this problem when we discuss a similar but somewhat simpler problem that arises in connection with the determination of optimum filters by the theory developed in the following sections.

Since linear systems form such an important class of systems in engineering, it is proper that we ask of any nonlinear theory, "How conveniently does this theory handle linear and nearly linear systems?" Although the Wiener theory includes within its scope linear as well as nonlinear systems, it is not particularly suited for application to the former. The reason for this can be seen by observing the form of the general Wiener system (Fig. 4). We note that the exponential function generator by-passes the Hermite polynomial generator. In order for the system of Fig. 4 to represent a linear system, the operation from the output of the Laguerre network to the output of the system must be linear. This means that the gain coefficients $A_{a}$ must have values that cause cancellation of the output of the exponential function generator and give the desired linear operation on the Laguerre coefficients. To achieve this cancellation effect, a very large number of Hermite functions will generally be required and, even then, we may have the unfavorable situation of obtaining a desired output that is the small difference of two large quantities. The nonlinear theory that is developed in the following sections does not suffer from this difficulty and, as we shall see, can be readily applied to linear and nearly linear systems, as well as to general nonlinear systems. 


\section{THE FILTER PROBLEM}

\subsection{OBJECTIVES AND ASSUMPTIONS}

We have seen how we can synthesize general nonlinear systems from a knowledge of their characterizing coefficients. We now turn our attention to the problem of deter mining optimum nonlinear systems or filters.

We shall deal with time-invariant nonlinear systems that operate on statistically stationary time functions. The filter problem considered here is one of determining that system, of a class of systems, that operates on the past of a given input time function $\mathrm{x}(\mathrm{t})$ to yield an output $\mathrm{y}(\mathrm{t})$ that best approximates a given desired output $\mathrm{z}(\mathrm{t})$ with respect to some error criterion. Wiener has shown that when the optimum filter is chosen from the class of linear systems, and when the mean-square-error criterion is adopted, this optimum filter is determined by the autocorrelation function of the input time function and the crosscorrelation function of the input with the desired output (7). Since these correlation functions determine the optimum mean-square linear filter, the same linear filter is optimum for all time functions that have these same correlation functions in spite of the fact that other statistical parameters of these time functions may be very different. It is in the search for better filters that we turn to nonlinear filters which make use of more statistical data than just first-order correlation functions.

As Zadeh (8) pointed out, there have been two distinct modes of approach to the optimum nonlinear filter problem. One approach parallels the approach of Wiener to linear systems by choosing the form or class of filters and then finding the optimum member of this class by minimizing the mean square error between the desired output and the actual system output. The other approach formulates an appropriate statistical criterion and then determines the optimum filter for this criterion with little or no restrictions upon the form of the filter. Both of these approaches yield equations for optimum filters in terms of higher-order statistics (higher-order distribution functions or correlation functions) of the input and desired output. In applying these approaches we are, in general, faced with two problems. First, we must obtain the necessary statistical data about the input and desired output and then we must solve the design equations, which usually are quite complex, for the optimum filter in terms of these data. In nonlinear filter problems we find that the amount of statistical data that are required in the design of the filter usually far exceeds the available data and we find it necessary to make certain simplifying assumptions or models of the signal and noise processes in order to calculate the required distributions.

Instead of assuming a statistical knowledge of the filter input and desired output, the approach to the nonlinear filter problem developed in this work assumes that we have at our disposal an ensemble member of the filter-input time function $x(t)$ and the corresponding ensemble member of the desired filter output $z(t)$. By recording or making direct use of a portion of the given filter-input time function, we obtain the 
ensemble member of $x(t)$. The ensemble member of $z(t)$ can be determined in different ways, depending upon the problem. For pure prediction problems $z(t)$ is obtained directly from $x(t)$ by a time shift. For filter problems involving the separation of signal from noise at the receiver in a communication link, we can, in the program for the design of the filter, record a portion of the desired signal $z(t)$ at the transmitter and the corresponding portion of $x(t)$ at the receiver. For the radar type of problem, in the program for the design of the filter, $z(t)$ can be generated corresponding to signals $x(t)$ received from known typical targets.

Since the ensembles of $x(t)$ and $z(t)$ contain all the statistical information concerning the filter input and desired output, and since we shall make direct use of these time functions in our filter determination, it is not necessary to make any assumptions about the distributions of $x(t)$ and $z(t)$. Thus, for example, in the problem of designing a filter to separate signal from noise, we need make no assumptions about the statistics of the signal or noise, or about how the two are mixed.

We note that, in most practical cases, our assumption of knowing a portion of $x(t)$ and $z(t)$ does not restrict us any more than the usual assumptions of knowing the higherorder probability densities of the input and desired output do; for at present, except in very simple cases, the only practical way of obtaining these statistics is to measure them from ensembles of $x(t)$ and $z(t)$ when these ensembles are available. When they are available, the present approach makes measurements on them that yield optimum filters directly, instead of first measuring the distributions and then solving design equations in terms of these measured values.

\subsection{RELATION TO THE CHARACTERIZATION PROBLEM}

The Wiener theory of nonlinear system characterization and synthesis provides us with a physically realizable operator on the past of a time function that includes within its scope a very large class of nonlinear systems. Consequently, we shall investigate the possibility of determining the optimum nonlinear filter (for a given task and a particular error criterion) from the class of systems of the Wiener theory.

Figure 3 shows the experimental procedure for obtaining the characterizing coefficients for a given nonlinear system (the system labeled "Nonlinear System Under Test"). Notice that the $\mathrm{A}_{a}$ are completely determined from a knowledge of the response of the given system to a shot-noise input. In fact, the presence of this system is not necessary in the experimental procedure of Fig. 3 if we have recordings of an ensemble member of the shot-noise input $x(t)$ and the corresponding output $y(t)$. By feeding the recording of $x(t)$ into the Laguerre network and the recording of $y(t)$ into the productaveraging device, in place of the output of the given system we obtain the $\mathrm{A}_{a}$ that correspond to the given system; that is, we obtain the $A_{a}$ that correspond to the system which operates on the shot noise $x(t)$ to yield $y(t)$. This arouses our curiosity concerning the possibility of determining the $A_{a}$ for the optimum filter problem directly from a knowledge of an ensemble member of its input and its desired output time 


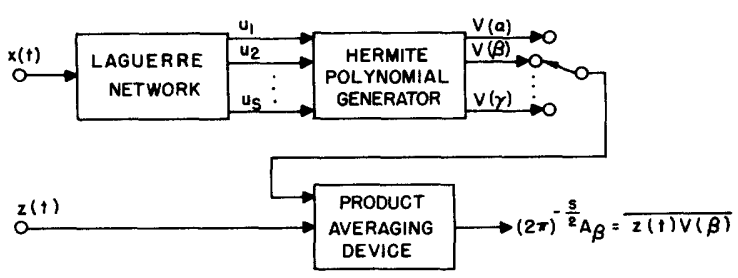

Fig. 7. Block diagram of experimental apparatus for the determination of an optimum filter when the given filter input $x(t)$ is shot noise. $z(t)$ is the desired filter output.

functions without actually having the filter at our disposal. To this end, let us consider the optimum filter problem and see how it differs from the characterization problem discussed above.

Unlike the characterization problem, in the determination of an optimum filter we do not have at our disposal the system labeled "Nonlinear System Under Test" in Fig. 3. In the filter problem this system would be the optimum filter - exactly what we are searching for. Consider the following problem. Suppose that we want to find a nonlinear filter whose input is a white gaussian time function $x(t)$ and whose desired output is the stationary random time function $z(t)$. Suppose also that we have at our disposal an ensemble member of $x(t)$ and the corresponding ensemble member of $z(t)$. We excite the Laguerre network of Fig. 3 with $x(t)$ and feed $z(t)$ into the product-averaging device in place of $y(t)$, as shown in Fig. 7. From the discussion above, it is clear that if the desired filter, which operates on $x(t)$ to yield $z(t)$, is a member of the class of systems considered in the Wiener theory, the test procedure of Fig. 7 will yield the $A_{a}$ that correspond to this system. We can then synthesize it in the general form shown in Fig. 4. However, it will usually happen that the desired system is not even physically realizable, let alone a member of the Wiener class of nonlinear systems. In this case the derivation of section 1.5 shows that the procedure indicated in Fig. 7 will yield that system of the Wiener class (with as many Laguerre coefficients and Hermite functions as are used in the test apparatus) whose output best approximates $z(t)$ in a weighted mean-square sense. Thus, for the special case of white gaussian filter input, we can adapt the Wiener method of characterization to the experimental determination of optimum nonlinear filters.

\subsection{NEED FOR A GENERAL ORTHOGONAL REPRESENTATION}

When the given filter input is not shot noise we can no longer apply the method described above to determine the optimum filter. Recall that the orthogonality relations that led to Eq. 26 for the $\mathrm{A}_{a}$ depended upon the fact that the Laguerre coefficients were gaussianly distributed and statistically independent, and this fact, in turn, depended on the fact that the input of the Laguerre network was shot noise. When $x(t)$ is not shot noise we no longer obtain independent relations (Eq. 26) for the $\mathrm{A}_{a}$ and the procedure 
for determining them (Fig. 7) is no longer valid. Thus we appreciate the need of an expression for a nonlinear operator in which the terms in its series representation are orthogonal in time, irrespective of the nature of the input time function. The development and application of such an operator is the subject of the following sections.

\section{OPTIMUM NONLINEAR FILTERS}

\subsection{OBJECTIVES}

We shall develop an orthogonal representation for nonlinear systems that enables the convenient determination of optimum nonlinear filters. The development is best described if, before proceeding to the general filter, we first examine the class of no-storage nonlinear filters.

\subsection{NO-STORAGE NONLINEAR FILTER}

By a no-storage system we mean one whose output, at any instant, is a unique function of the value of its input at the same instant. We call the input-output characteristic of this system the transfer characteristic.

Let $x(t)$ and $z(t)$ be the given filter-input and desired filter-output time functions, respectively. We assume that $x(t)$ and $z(t)$ are bounded, continuous time functions. This is clearly no restriction in the practical case and it enables us to confine our attention to approximating desired filter transfer characteristics that are bounded and continuous. Since $x(t)$ is bounded, there exists an $a$ and $b$ which are such that $a \leqslant x(t) \leqslant b$ for all $t$. Now consider a set of $n$ functions $\phi_{j}(x) \quad(j=1, \ldots, n)$ over the interval $(a, b)$. These functions are defined as follows:

$$
\phi_{j}(x)=\left\{\begin{array}{lll}
1 \text { for } x_{j}-\frac{w}{2} \leqslant x<x_{j}+\frac{w}{2}, & j=(1, \ldots, n-1) & \\
\text { and } x_{j}-\frac{w}{2} \leqslant x \leqslant b, & j=n & w=\frac{b-a}{n} \\
0 \text { for all other } x & x_{j}=a+w\left(j-\frac{1}{2}\right)
\end{array}\right.
$$

A plot of the $j^{\text {th }}$ function of this set of functions is shown in Fig. 8. [A separate

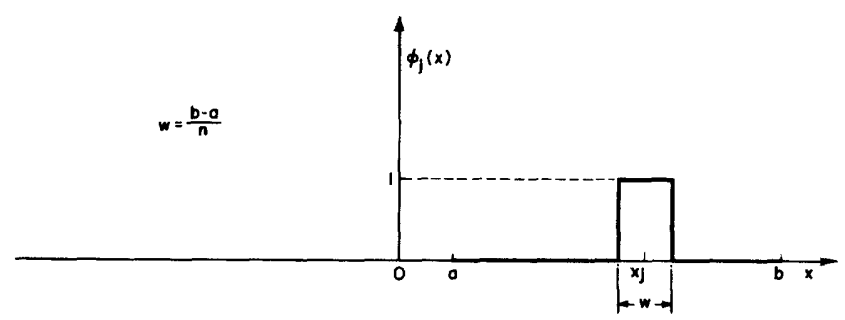

Fig. 8. Gate function $\phi_{j}(x)$. 
definition is given for $\phi_{n}(x)$ in order to include the point $b$. In practical application we simply generate $\mathrm{n}$ functions of equal width that cover the interval (a,b).] Clearly, this set of functions is normal and orthogonal over the interval $(a, b)$. We shall refer to these functions as "gate functions." Let us define $\mathrm{y}$ as a gate-function expansion of $\mathrm{x}$,

$$
y=\sum_{j=1}^{n} a_{j} \phi_{j}(x)
$$

It is clear that by taking $n$ sufficiently large y can be made to approximate any singlevalued continuous function of $x$ arbitrarily closely everywhere on the interval $(a, b)$.

When $\mathrm{x}$ is a function of time it is convenient to write Eq. 38 as

$$
y(t)=\sum_{j=1}^{n} a_{j} \phi_{j}[x(t)]
$$

As a consequence of the nonoverlapping property of the gate functions along the $\mathrm{x}$-axis, the $\phi_{j}[x(t)]$ will, for any single-valued time function $x(t)$, form an orthogonal set in time, as well as an orthonormal set in $x$. Furthermore, this time-domain orthogonality holds for any bounded weighting function $G(t)$. That is,

$$
G(t) \phi_{j}[x(t)] \phi_{k}[x(t)]= \begin{cases}0 & j \neq k \\ G(t) \phi_{j}^{2}[x(t)] & j=k\end{cases}
$$

Relation 39 specifies the form of an equation that defines a no-storage nonlinear system. The determination of an optimum no-storage filter for a given error criterion consists of choosing the $a_{j}$ in such a manner that, for a given $x(t)$, the error between $y(t)$ and the desired output $z(t)$ is a minimum. We adopt a weighted mean-square-error criterion in which $G(t)$ is, as we shall discuss later, a non-negative weighting function at our disposal. More specifically, we minimize the error

$$
\mathscr{E}=\lim _{T \rightarrow \infty} \frac{1}{2 T} \int_{-T}^{T} G(t)\left\{z(t)-\sum_{j=1}^{n} a_{j} \phi_{j}[x(t)]\right\}^{2} d t
$$

with respect to the $n$-coefficients $a_{j}$. Differentiating with respect to $a_{k}$ and setting the result to zero, we obtain

$$
\frac{\partial \mathscr{E}}{\partial a_{k}}=\lim _{T \rightarrow \infty} \frac{1}{2 T} \int_{-T}^{T}-2 G(t) \phi_{k}[x(t)]\left\{z(t)-\sum_{j=1}^{n} a_{j} \phi_{j}[x(t)]\right\} d t=0
$$

where $k=(1, \ldots, n)$. If we denote the operation of time-averaging by a bar over the 
averaged variable, Eq. 42 can be written as

$$
G(t) \phi_{k}[x(t)] \sum_{j=1}^{n} a_{j} \phi_{j}[x(t)]=\overline{z(t) G(t) \phi_{k}[x(t)]}
$$

If we make use of the time-domain orthogonality of the gate functions in Eq. 40, Eq. 43 reduces to

$$
a_{k} \overline{G(t) \phi_{k}^{2}[x(t)]}=\overline{z(t) G(t) \phi_{k}[x(t)]}
$$

It follows from the definition of the $\phi_{j}(x)$ given in Eq. 37 that $\phi_{j}^{2}[x(t)]=\phi_{j}[x(t)]$, so that Eq. 44 is equivalent to the equation

$$
a_{k} \overline{G(t) \phi_{k}[x(t)]}=\overline{z(t) G(t) \phi_{k}[x(t)]}
$$

This equation provides a convenient experimental means of determining the desired coefficients $a_{k}$. The experimental procedure for the evaluation of these coefficients is shown in Fig. 9. An ensemble member of $x(t)$ is fed into a level-selector circuit and the corresponding ensemble member of $z(t)$ is fed into the product-averaging device. The output of the level-selector circuit is unity whenever the amplitude of $x(t)$ falls within the interval of the $\mathrm{k}^{\text {th }}$ gate function and zero at all other times. This output is used to gate the weighting function $G(t)$. The output of the gate circuit is then averaged and also multiplied by $z(t)$ and averaged to yield the two quantities necessary for determining $a_{k}$ in Eq. 45.

From a knowledge of the $\mathrm{a}_{\mathrm{k}}$ we can directly construct a stepwise approximation, like that of Fig. 10, to the desired optimum transfer characteristic (see Eq. 38). The synthesis of the filter can be carried out formally in accordance with Eq. 38 by using level-selector circuits and an adder, as shown in Fig. 11, or we can synthesize the optimum characteristic by any of the other available techniques, such as piecewiselinear approximations or function generators.

In order to become more familiar with the operation and terminology of this method, let us consider a very simple example. We shall do analytically what, in practice, the experimental procedure that is indicated in Fig. 9 does for us.

EXAMPLE 2. Suppose we are given an ensemble member of $x(t)$ and the corresponding ensemble member of $z(t)$. Furthermore, suppose that the desired filter output $z(t)$ is equal to $f[x(t)]$, where $f$ is a continuous real function of $x$. We desire to verify the fact that the filter determined by the procedure indicated in Fig. 9 is actually a stepwise approximation to the transfer characteristic $f(x)$. For simplicity, let us assume that $n$ has been chosen sufficiently large so that the function $f(x)$ is approximately constant over the width of the gate functions and let us choose $G(t)$ equal to a constant so that the conventional mean-square-error criterion will result. For these conditions, whenever $\phi_{k}[x(t)]$ has a nonzero value, $x$ must lie in the interval of width $w$ 


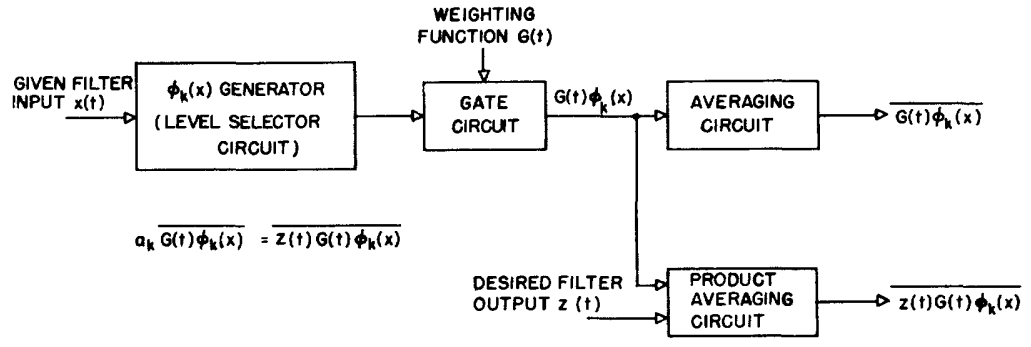

Fig. 9. Experimental procedure for the determination of the optimum filter coefficients for no-storage filters. $x(t)$ and $z(t)$ are corresponding ensemble members of the stationary input and desired output time functions.

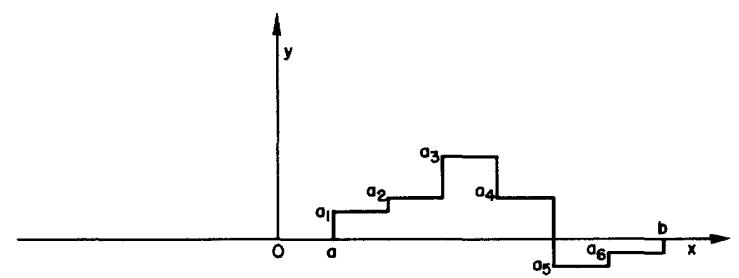

Fig. 10. Example of a stepwise representation of a transfer characteristic over the interval $(a, b)$. The $a_{k}$ are the optimum filter coefficients evaluated by the procedure indicated in Fig. 9.

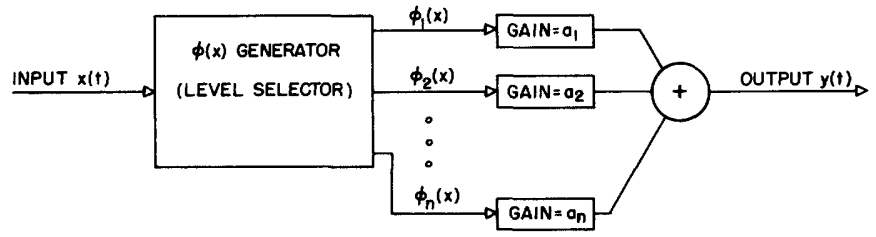

Fig. 11. Example of the formal synthesis of no-storage filters in accordance with Eq. 38 .

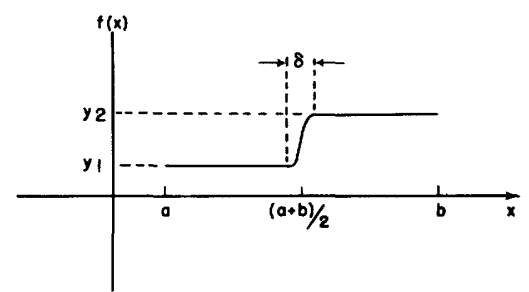

Fig. 12. A transfer characteristic of a no-storage nonlinear system. 
about $x_{k}$, and $z(t)$ is approximately equal to $f\left(x_{k}\right)$. Equation 45 then becomes

$$
a_{k} \overline{\phi_{k}[x(t)]} \approx f\left(x_{k}\right) \overline{\phi_{k}[x(t)]}
$$

from which we obtain the relation

$$
\mathrm{a}_{\mathrm{k}} \approx \mathrm{f}\left(\mathrm{x}_{\mathrm{k}}\right)
$$

for the $a_{k}$ which shows (see Eq. 38) that they determine a filter that is a stepwise approximation to the desired transfer characteristic $f(x)$. [A closer examination of example 2 shows that the same results can be obtained for any weighting function $G(t)$. This is because the desired filter, in this example, is a member of the class of nostorage filters and, therefore, as $\mathrm{n} \rightarrow \infty$ the error $\mathscr{E}$ in Eq. 41 can be made zero for any $G(t)$.

In addition to knowing that as $n \rightarrow \infty$ the gate-function expansion (Eq. 38) can approximate any continuous transfer function arbitrarily closely, it is of practical interest to investigate how the expansion converges for small $\mathrm{n}$ as $\mathrm{n}$ is increased when the coefficients are so chosen that they minimize the mean square error. This is most easily done with the aid of an example.

EXAMPLE 3. The transfer characteristic of Fig. 12 is the one that we desire to approximate. The simplest gate-function expansion is that for which $\mathrm{n}=1$. The best mean-square approximation occurs for $a_{1}=\left(y_{1}+y_{2}\right) / 2$. For $n=2$, the best approximation occurs for $\mathrm{a}_{1}=\mathrm{y}_{1}$ and $\mathrm{a}_{2}=\mathrm{y}_{2}$. This approximation is considerably better than that for $n=1$. Now consider $n=3$. The best mean-square approximation is, by inspection, $a_{1}=y_{1}, a_{2}=\left(y_{1}+y_{2}\right) / 2$ and $a_{3}=y_{2}$. But this is a worse approximation than that for $\mathrm{n}=2$ ! For $\mathrm{n}=4$, clearly, we must do at least as well as for $\mathrm{n}=2$, since $\mathrm{a}_{1}=\mathrm{a}_{2}=\mathrm{y}_{1}$ and $a_{3}=a_{4}=y_{2}$ constitute a possible solution. Again, for this example, the approximation for $n=5$ is inferior to that for $n=2$ or $n=4$ but better than the $n=3$ approximation. A moment's reflection reveals that the reason for this peculiar convergence is that the function $f(x)$ changes appreciably in an interval that is small compared with the width of the gate functions and thus the position of the gate functions along the $x-$ axis is critical. For this example, when $\mathrm{n}$ is even, one gate function ends at $x=(a+b) / 2$ and another begins, thus providing a nice fit to $f(x)$. For $n$ odd, one gate function straddles the point $x=(a+b) / 2$ and, because of symmetry, it will have a coefficient equal to $\left(y_{1}+y_{2}\right) / 2$. As we increase $n$ beyond the point at which the width $(w=(b-a) / n)$ of the gate functions becomes less than $\delta$, the position of the gate functions become less and less critical, the oscillatory behavior disappears and the expansion converges to $f(x)$ everywhere.

From this simple example, we can draw some general conclusions regarding the convergence of the gate-function expansion to continuous functions. When the desired function changes appreciably in an interval of $\mathrm{x}$ that is comparable to or smaller than $\mathrm{w}$, it may happen that an increase in $\mathrm{n}$ will result in a poorer approximation. However, 
if $\mathrm{n}$ is increased by an integral factor, the approximation will always be at least as good as it was before the increase. Furthermore, if $\mathrm{n}$ is taken large enough so that the function is essentially constant over any interval of width $\mathrm{w}$, then any increase in $\mathrm{n}$ will yield at least as good an approximation as the one before the increase. Thus, in the practical application of this theory, if we increase $\mathrm{n}$ and get an inferior filter we should not be alarmed. It is merely an indication that the desired filter characteristic has a large slope over some interval. By further increasing $\mathrm{n}$ the desired characteristic will be obtained.

We have assumed, for the sake of convenience, that each gate function had the same width $\mathrm{w}$. But this is not a necessary restriction. It is sufficient to choose them so that they cover the interval $(a, b)$ and do not overlap. Thus, if we have some a priori knowledge about the optimum transfer characteristic, we may be able to save time and work in determining it by judiciously choosing the widths of the $\phi_{j}(x)$. In fact, after evaluating any number $m$ of the $a_{k}$ we are free to alter the widths of the remaining functions $\phi_{j}(x)(j>m)$ as we proceed. This flexibility is permissible because, in taking advantage of it, we do not disturb the time-domain orthogonality of the gate functions.

\subsection{LINEAR AND NONLINEAR SYSTEMS FROM THE POINT OF VIEW OF FUNCTION SPACE}

In section 1.3 we saw how we can characterize the past of a time function by the coefficients of a complete set of orthogonal functions such as the Laguerre functions. Let us now think of a function space which has for its basis the Laguerre functions. Just as in a vector space a given vector can be represented as a linear combination of the basis vectors, so in function space a given function (satisfying appropriate regularity conditions) can be represented as a linear combination of the functions that form the basis of the space. We can think of the Laguerre coefficients of a function $x(t)$ as being the scalar components of $x(t)$ along the respective basis vectors. At any instant, the past of $x(t)$ is represented by the point in function space that corresponds to the tip of the vector whose scalar components are the Laguerre coefficients of the past of $x(t)$.

We have also found that any function of the past of $x(t)$ can be expressed as a function of the Laguerre coefficients of this past. In terms of the function space, then, a function of the past of $x(t)$ can be expressed as a function of position in this space. We can say that we generate the desired function of the past of $x(t)$ by a transformation that maps the function space on a line - the line corresponds to the amplitude of the desired function. This concept provides a powerful tool in the study of linear and nonlinear systems. To better understand it, let us consider the Wiener theory in the light of this concept. The output of the general Wiener nonlinear system is expressed (Eq. 17) as a Hermite-function expansion of the Laguerre coefficients of the past of the input time function. The Laguerre functions form the basis of the function space of the past of the input and the Hermite-function expansion maps this space on a line - the amplitude of the system output. 
Several important concepts follow immediately from this viewpoint. The first, which was made evident by the Wiener theory, is that any system (of the broad class considered in the Wiener theory) can be represented by the cascade of a linear system followed by a no-memory nonlinear system. The outputs of the linear system characterize the past of the input as a point in function space and the no-memory nonlinear system maps this space onto a line. Second, we see that in principle (we assume that the complete set of Laguerre functions is used) the difference between any two systems is accounted for by a difference in the no-memory part that performs the mapping. For example, if the mapping is linear (we shall discuss this case in a later section), then a linear system is represented; if it is not, then a nonlinear system is represented. Since the difference between two systems is just in this mapping, the problem of determining an optimum system for a desired performance and given error criterion becomes one of determining the optimum no-memory system that maps the function space on the output.

Finally, we see that this function-space point of view provides the key for finding a general orthogonal expansion for the output of a nonlinear system. For reasons that will become evident in the next section, we desire to obtain a series expansion for the output of a nonlinear system in which the terms are mutually orthogonal in time. Furthermore, we require that this orthogonality be independent of the input time function. This is achieved by choosing a mapping that partitions the function space into nonoverlapping cells and by letting each term in the series expansion represent the system output for a particular cell in the function space. Since at any instant the past of the input is represented by only one point in the function space, only one term in the series expansion will be nonzero at any instant; thus all the terms are mutually orthogonal in time. The gate-function expansion for the no-storage filter (Eq. 39) is recognized to be an application of this approach in the simple case for which the input space is just a line. We shall apply this approach to the more general case of a finite dimensional space. (Although the function space of which we have spoken is infinite dimensional, we shall continue to use the term even when we speak of a finite number of Laguerre functions.)

\subsection{GENERAL NONLINEAR FILTER WITH STORAGE}

The class of nonlinear systems now considered is the same as that of the Wiener theory. Without introducing any physical restriction, we shall, for convenience, assume that the given filter input $x(t)$ is bounded. As in the Wiener theory, the past of $x(t)$ is characterized by its Laguerre coefficients. It can be easily shown that these coefficients are bounded if $x(t)$ is bounded. Recall that the Laguerre coefficients of $x(t)$ are given by the convolution of $x(t)$ with the Laguerre functions. That is,

$$
u_{n}(t)=\int_{0}^{\infty} x(t-\tau) h_{n}(\tau) d \tau
$$


where $h_{n}$ is defined as in Eq. 1. It follows that

$$
\left|u_{n}(t)\right| \leqslant \int_{0}^{\infty}|x(t-\tau)|\left|h_{n}(\tau)\right| d \tau
$$

Assume $|x(t)| \leqslant M$ for all $t$. Then

$$
\left|u_{n}(t)\right| \leqslant M \int_{0}^{\infty}\left|h_{n}(\tau)\right| d \tau
$$

But, from Eq. 1, we see that the Laguerre functions are polynomials multiplied by decaying exponentials; hence they are absolutely integrable. Thus the Laguerre coefficients are bounded if $\mathrm{x}(\mathrm{t})$ is bounded.

Now consider the function space formed by $s$ Laguerre coefficients. We divide the bounded region over which each Laguerre coefficient ranges into $\mathrm{n}$ intervals and define a set of gate functions, as in Eq. 37, for each coefficient. (It is only for convenience in notation that we choose the same number of gate functions for each Laguerre coefficient.) We have seen that if we choose an expansion of these coefficients that partitions this function space into nonoverlapping cells and is such that each term in the expansion represents the system output for one cell in the function space, an orthogonal expansion is obtained. To this end, consider the expansion

$$
y(t)=\sum_{i=1}^{n} \sum_{j=1}^{n} \ldots \sum_{h=1}^{n} a_{i, j} \ldots, h \phi_{i}\left(u_{1}\right) \phi_{j}\left(u_{2}\right) \ldots \phi_{h}\left(u_{s}\right)
$$

in which the $\phi^{\prime} s$ are the gate functions defined in Eq. 37. Let us examine a typical term in this expansion. The term

$$
a_{i, j} \ldots, h \phi_{i}\left(u_{1}\right) \phi_{j}\left(u_{2}\right) \ldots \phi_{h}\left(u_{s}\right)
$$

is nonzero only when the amplitude of $u_{1}$ is in the interval for which $\phi_{i}\left(u_{1}\right)$ is unity and the amplitude of $u_{2}$ is in the interval for which $\phi_{j}\left(u_{2}\right)$ is unity, and so on for each Laguerre coefficient. The collection of these intervals defines a cell in the function space and thus the term in Eq. 52 is nonzero only when this cell is occupied. Hence the expansion (Eq. 51) divides the function space into nonoverlapping cells and each term represents $y$ when the corresponding cell in the function space of the input is occupied. Thus the terms are mutually orthogonal in time for any $x(t)$. It is clear that as the width of the gate functions is decreased, by increasing $n$, the cells become smaller and y can be made to approximate any continuous function of the $u$ 's everywhere with vanishing error.

If $\Phi(a)$ represents the function $\phi_{i}\left(u_{1}\right) \phi_{j}\left(u_{2}\right) \ldots \phi_{h}\left(u_{s}\right)$ and $A_{a}$ represents the corresponding coefficient $a_{i, j}, \ldots, h, E q .51$ takes the simplified form 


$$
y(t)=\sum_{a} A_{a} \Phi(a)
$$

This equation is the desired orthogonal representation for nonlinear systems involving storage. We now proceed to determine the $\mathrm{A}_{a}$ for the optimum filter problem. As in the case of the no-storage filter (Eq. 41), we adopt a weighted mean-square-error criterion and minimize the error

$$
\mathscr{E}=\lim _{\mathrm{T} \rightarrow \infty} \frac{1}{2 \mathrm{~T}} \int_{-\mathrm{T}}^{\mathrm{T}} \mathrm{G}(\mathrm{t})\left\{\mathrm{z}(\mathrm{t})-\sum_{a} \mathrm{~A}_{a} \Phi(a)\right\}^{2} \mathrm{dt}
$$

with respect to the coefficients $A_{a}$. For the coefficient $A_{\beta}$, we have

$$
\frac{\partial \mathscr{E}}{\partial \mathrm{A}_{\beta}}=\lim _{\mathrm{T} \rightarrow \infty} \frac{1}{2 \mathrm{~T}} \int_{-\mathrm{T}}^{\mathrm{T}}-2 \mathrm{G}(\mathrm{t}) \Phi(\beta)\left\{\mathrm{z}(\mathrm{t})-\sum_{a} \mathrm{~A}_{a} \Phi(a)\right\} d t
$$

For the error to be a minimum with respect to $A_{\beta}$, we set this equation to zero. The result is

$$
\overline{G(t) \Phi(\beta) \sum_{a} A_{a} \Phi(a)}=\overline{z(t) G(t) \Phi(\beta)}
$$

If advantage is taken of the time-domain orthogonality of the $\Phi^{\prime} s$, this equation reduces to

$$
A_{\beta} \overline{G(t) \Phi^{2}(\beta)}=\overline{z(t) G(t) \Phi(\beta)}
$$

Since the $\Phi^{\prime} s$ are products of gate functions, they can only take on the values zero or unity; hence Eq. 57 is equivalent to

$$
A_{\beta} \overline{G(t) \Phi(\beta)}=\overline{z(t) G(t) \Phi(\beta)}
$$

which forms the basis of the experimental procedure for determining the optimum filter coefficients.

The apparatus for the determination of the optimum filter coefficients is shown in Fig. 13. An ensemble member of $x(t)$ is fed into the Laguerre network and the corresponding ensemble member of $z(t)$ is fed into the product-averaging device. The outputs of the Laguerre network are fed into a no-memory nonlinear circuit consisting of level selectors and gate or coincidence circuits. This circuit generates the $\Phi^{\prime} s$. Since the $\Phi^{\prime} s$ are either zero or unity, they can be multiplied by $G(t)$ in a simple gate circuit. The output of this gate circuit is averaged and also multiplied by $z(t)$ and averaged to yield the two quantities that are necessary to find the optimum coefficients in accordance with Eq. 58. 


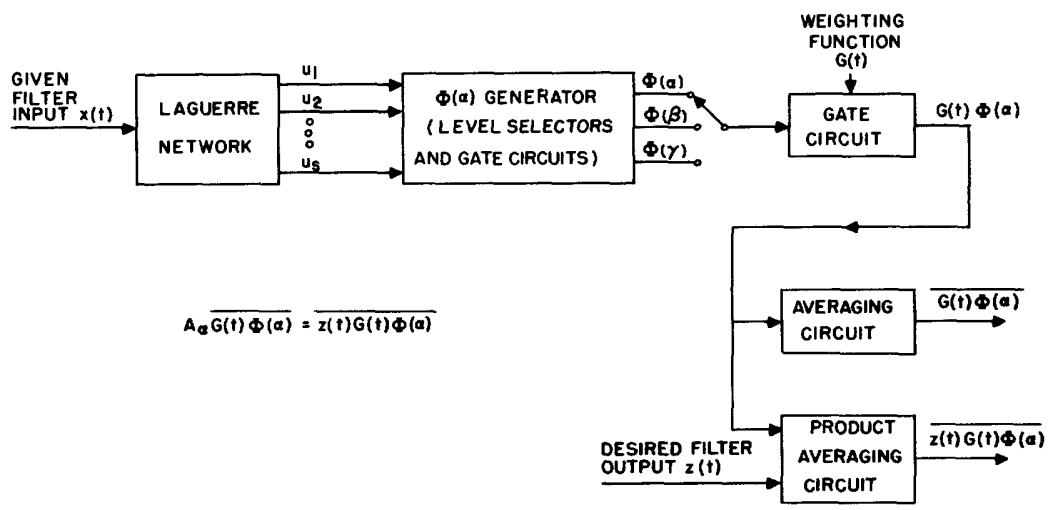

Fig. 13. Experimental procedure for the determination of optimum nonlinear filters involving storage.

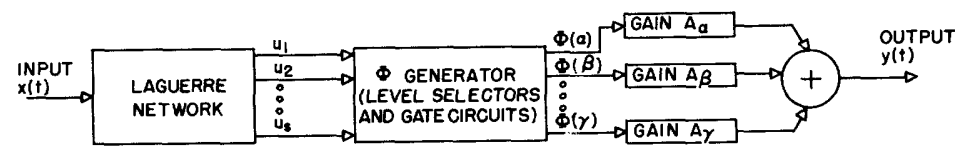

Fig. 14. Synthesis of general optimum nonlinear filters in accordance with Eq. 53 .
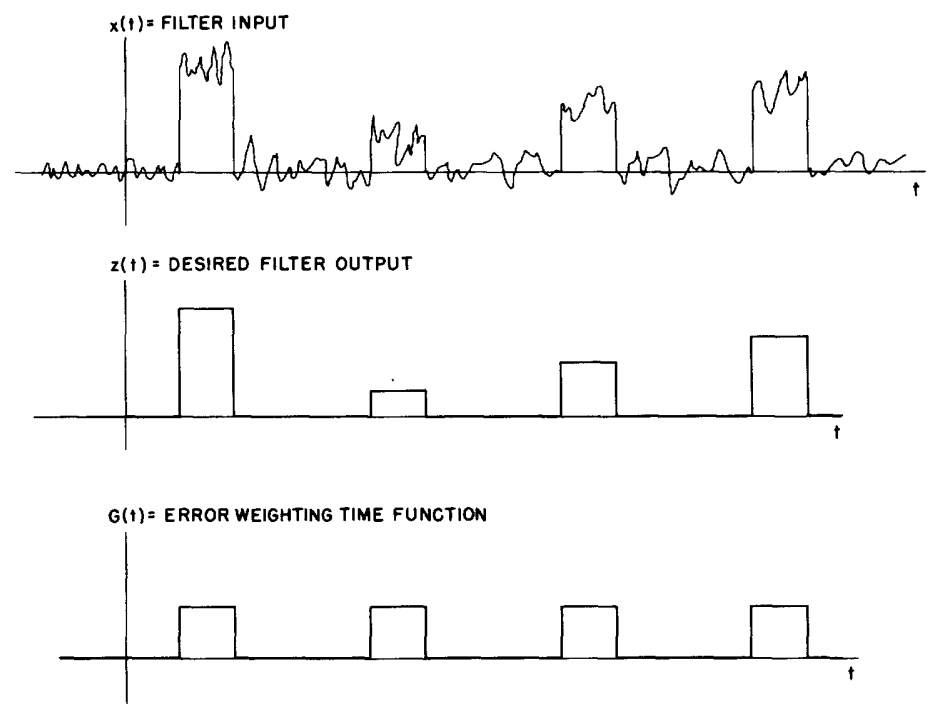

Fig. 15. Example of the use of the error weighting time function. 
After the optimum coefficients have been determined, the nonlinear system can be synthesized formally in accordance with Eq. 53, as indicated in Fig. 14. In Fig. 14 we note that the operation from the outputs of the Laguerre network to the system output $y(t)$ is a no-memory operation. That is, $y$ is an instantaneous function of the Laguerre coefficients. Once the A's are known, this function is directly specified and any other method of synthesizing no-storage systems for a prescribed operation can be used. One such method is described in reference 9.

In the procedure described above for determining and synthesizing optimum nonlinear filters the use of gate functions in the expansion of Eq. 51 is of central importance. Let us examine some of the consequences of this:

1. The use of gate functions provides us with a series representation for the output of the filter in which the time-domain orthogonality of the terms of the series is independent of the filter input. This enables us to obtain the optimum filter coefficients for arbitrary filter inputs without solving simultaneous equations.

2. Since the gate functions are orthogonal with respect to any weighting factor, we can determine optimum filters for weighted mean-square-error criteria.

3. In most series representations of a function we encounter the difficulty that over some region of the independent variable small differences of two or more large terms are necessary to represent the desired function. In the gate-function expansion (Eq. 53) only one term has a nonzero value at any one instant of time; so this difficulty does not arise.

4. In general, expansions that represent nonlinear functions involve the use of multipliers in the experimental circuits. (For example, if a Taylor series or Hermite function expansion is used.) The use of gate functions replaces the multipliers by simpler level selectors and coincidence circuits.

\subsection{ERROR CRITERION}

An error weighting function $G(t)$ appears in the error expressions Eqs. 41 and 54 for the no-storage and the general filter. The choice of this weighting function will, of course, depend upon the particular problem. It may be chosen as a function of the past, present, and/or future of $x(t)$ and $z(t)$ and can be generated in the laboratory from the recorded ensemble members of $x(t)$ and $z(t)$. If $G(t)$ is a constant, then the meansquare-error criterion results. Other choices for $G(t)$ enable us to design filters for different error criteria and to introduce a priori information into the filter design. In this section a few choices of $G(t)$ are discussed. We restrict $G(t)$ to be non-negative, since the concept of negative error is not meaningful.

One choice of $G(t)$ is illustrated by the following example. Let the signal componento $z(t)$ of the filter input $x(t)$ consist of amplitude-modulated pulses that occur periodically. As shown in Fig. 15, $x(t)$ is $z(t)$ corrupted in some way by noise. We assume that we know when the signal pulses occur. Our object is to determine their amplitude. The optimum mean-square filter, of a given class of filters, for recovering $z(t)$ from $x(t)$ 
is the one for which the time average of $[z(t)-y(t)]^{2}$ is a minimum for all filters of the class. (In this expression $y(t)$ is the filter output.) However, we are actually interested in minimizing the error between $z(t)$ and $y(t)$ only during the time when signal pulses are present. By choosing a $G(t)$ that is a constant during the time intervals when signal pulses occur and zero at all other times (Fig. 15) we can design just such a filter. In general, if both of these filters have the same degree of freedom (i.e., the same number of Laguerre coefficients and gate functions) the performance of the one designed with the weighting function will be superior to that of the mean-square filter, since all the freedom of the former is used to minimize the error over the time intervals of interest. Thus, through $\mathrm{G}(\mathrm{t})$, we have introduced a priori information (about the periodic occurrence of the signal pulses) into the filter design in order to obtain a better filter.

In other problems it may be desirable to choose $G(t)$ to be a function of the magnitude of the difference between the present values of $x(t)$ and $z(t)$ so that the freedom of the filter is used to do a better job, on the average, when $|x(t)-z(t)|$ lies in certain ranges, at the expense of its performance when this difference lies in other ranges. For example, we might desire that the filter output be as close as possible to the desired output, on the average, when the difference between the input and desired output is small and, when this difference is large, we might choose to attach less significance to the filter output. In such a case we could let $G(t)$ be $|x(t)-z(t)|^{-n}$. For large $n$, this $G(t)$ weights small errors between $x(t)$ and $z(t)$ much more heavily than large errors.

The choices of $G(t)$ are limited only by the ingenuity of the designer in making the best use of the data. By precisely defining the object of the particular filter problem and carefully studying the nature of the problem, he may often be able to choose a $G(t)$ that yields a far better filter than the mean-square filter.

\subsection{MINIMUM-ERROR DETERMINATION}

Paralleling the Wiener approach to linear filters, we shall find an expression for the minimum error of nonlinear filters that can be evaluated from a knowledge of the input and desired output time functions. The general expression for the error between the desired output and the actual nonlinear system output is given by Eq. 54. We have seen from Eq. 55 that, for this error to be a minimum with respect to the $A_{a}$, we must have

$$
\lim _{T \rightarrow \infty} \frac{1}{2 T} \int_{-T}^{T} G(t) \Phi(\beta)\left[z(t)-\sum_{a} A_{a} \Phi(a)\right] d t=0 \quad \text { for all } \beta
$$

hence

$$
\lim _{T \rightarrow \infty} \frac{1}{2 T} \int_{-T}^{T} \sum_{\beta} A_{\beta} \Phi(\beta) G(t)\left[z(t)-\sum_{a} A_{a} \Phi(a)\right] d t=0
$$

Now Eq. 54 can be written as follows: 


$$
\mathscr{E}=\overline{G(t) z(t)\left[z(t)-\sum_{a} A_{a} \Phi(a)\right]}-\overline{\sum_{\beta} A_{\beta} \Phi(\beta) G(t)\left[z(t)-\sum_{a} A_{a} \Phi(a)\right]}
$$

But, from Eq. 60, we see that the term on the right side of Eq. 61 is zero for the optimum filter. Using this fact and inserting the expression for the optimum filter coefficients (Eq. 58) in Eq. 61, we obtain the desired expression for the minimum error.

$$
\mathscr{E}_{\min }=\overline{z^{2}(t) G(t)}-\sum_{a} \frac{\overline{z(t) G(t) \Phi(a)}}{\overline{G(t) \Phi(a)}}
$$

This equation expresses the error of the optimum system with a given number of Laguerre coefficients and gate functions in terms of the filter-input and desired output time functions. If, in Eq. $62, \Phi(a)$ is changed to $\phi_{j}(x)$ and the summation is taken over $j$, then we have the minimum-error expression for no-storage filters.

With the addition of a squaring device at the output of the product-averaging circuit in Fig. 13 the quantities necessary to determine $\mathscr{E}_{\min }$ can be evaluated and $\mathscr{E}_{\text {min }}$ can thus be found without first constructing the optimum filter. Similar apparatus could be built to evaluate $\mathscr{E}_{\min }$ automatically upon application of $x(t)$ and $z(t)$. For those filters that have a sufficiently small number of $A_{a}$ (for example, no-storage filters and simple filters involving one or two Laguerre coefficients) all the terms in the sum (Eq. 62) could be evaluated simultaneously and added. This would give a rapid way of finding $\mathscr{E}_{\mathrm{min}}$. When the number of coefficients becomes very large, then, in order to save equipment at the expense of time, the terms in the sum could be evaluated sequentially. This apparatus would be useful in deciding a priori the complexity of the nonlinear filter to use for a particular problem. It would also enable us to decide whether or not it is worth while to construct a complicated nonlinear filter to replace a simple linear or nonlinear one. Since such apparatus would make use of the same measurements that determine the $A_{\alpha}$, if after measuring its error we decided to build the filter, we could construct it without further measurements.

\subsection{THE STATISTICAL APPROACH}

We can shed additional light on the filter theory that was developed in the previous sections by formulating the same problem on a statistical basis. As before, we shall characterize the past of the filter input by $s$ Laguerre coefficients $u_{1}, \ldots, u_{s}$ and determine the optimum nonlinear operator that relates these coefficients to the system output for a weighted mean-square-error criterion.

Consider an ensemble of the Laguerre coefficients $u_{1}(t), \ldots, u_{s}(t)$ and corresponding ensembles of the system output $y(t)$, the desired output $z(t)$, and the weighting function $G(t)$. We shall regard $u_{1}, \ldots, u_{s}, y, z$, and $G$ as random variables. We want to find the $y$, as a function of the $u^{\prime} s$, that minimizes the error 
$\mathscr{E}=\int_{G} \int_{z} \int_{u_{1}} \ldots \int_{u_{S}} G(z-y)^{2} P\left(z, G, u_{1}, \ldots, u_{s}\right) d u_{1} \ldots d u_{s} d z d G$

This expression is seen to correspond to the weighted mean-square-error criterion of Eq. 54. The y that minimizes this expression is found by direct application of the calculus of variations. Setting the variation of $\mathscr{E}$ to zero, we obtain

$\delta(\mathscr{E})=\int_{\mathrm{G}} \int_{\mathrm{z}} \int_{\mathrm{u}_{1}} \ldots \int_{\mathrm{u}_{\mathrm{S}}}-2 \mathrm{G}(\mathrm{z}-\mathrm{y}) \mathrm{P}\left(\mathrm{z}, \mathrm{G}, \mathrm{u}_{1}, \ldots, \mathrm{u}_{\mathrm{s}}\right) \delta(\mathrm{y}) \mathrm{du}_{1} \ldots \mathrm{du}_{\mathrm{s}} \mathrm{dz} \mathrm{dG}=0$

or the equivalent expression

$$
\begin{aligned}
\delta(\mathscr{E})= & \int_{\mathrm{G}} \int_{\mathrm{z}} \int_{\mathrm{u}_{1}} \ldots \int_{\mathrm{u}_{\mathrm{s}}}-2 \mathrm{G}(\mathrm{z}-\mathrm{y}) \mathrm{P}\left(\mathrm{z}, \mathrm{G} \mid \mathrm{u}_{1}, \ldots, \mathrm{u}_{\mathrm{s}}\right) \mathrm{dz} \mathrm{dG} \delta(\mathrm{y}) \mathrm{P}\left(\mathrm{u}_{1}, \ldots, \mathrm{u}_{\mathrm{s}}\right) \\
& \times \mathrm{du}_{1} \ldots \mathrm{du}_{\mathrm{s}}=0
\end{aligned}
$$

which must be true for all $\delta(y)$, where $\delta(y)$ is the variation in y. Equation 65 will hold for all $\delta(y)$ if we set

$$
\int_{G} \int_{z} G(z-y) P\left(z, G \mid u_{1}, \ldots, u_{s}\right) d z d G=0
$$

From this equation, we obtain the equation

$y \int_{G} \int_{z} G P\left(z, G \mid u_{1}, \ldots, u_{s}\right) d z d G=\int_{G} \int_{z} G z P\left(z, G \mid u_{1}, \ldots, u_{s}\right) d z d G$

Performing the integration with respect to $z$ in the left side of Eq. 67, we obtain

$$
y=\frac{\int_{G} \int_{z} G z P\left(z, G \mid u_{1}, \ldots, u_{s}\right) d z d G}{\int_{G} G P\left(G \mid u_{1}, \ldots, u_{s}\right) d G}
$$

This equation, although it is certainly not very suggestive of a filter design, is the desired relation between the optimum filter output and the s Laguerre coefficients of the past of the filter input. It should be noted that in deriving Eq. 68 no restrictions were made on the relationship between $y$ and the $s$ Laguerre coefficients; hence this equation yields the optimum $y$ in terms of these coefficients. Equation 68 takes on the more familiar form 


$$
y=\int_{z} z P\left(z \mid u_{1}, \ldots, u_{s}\right) d z
$$

when $\mathrm{G}$ is a constant, corresponding to the mean-square-error criterion. In this case we have the result that the optimum output for a given past of the input is just the con ditional mean of the desired output, given this past of the input.

Let us now investigate the relation between the result of the statistical approach (Eq. 68) and that of the so-called time-domain approach (Eq. 58). We shall express both of the time averages in Eq. 58 as ensemble averages and then compare the result with Eq. 68. The average on the left side of Eq. 58 is equal to

$$
\overline{G(t) \Phi(a)}=\int_{G} \int_{u_{1}} \ldots \int_{u_{2}} G \Phi(a) P\left(G \mid u_{1}, \ldots, u_{s}\right) P\left(u_{1}, \ldots, u_{s}\right) d G d u_{1} \ldots d u_{s}
$$

For the present, let us assume that the width of the gate functions is so small that the $u^{\prime}$ 's are essentially constant over each cell in the function space. Then Eq. 70 can be written

$$
\overline{G(t) \Phi(\alpha)} \approx P\left(u_{1_{j}}, \ldots, u_{s_{h}}\right)\left(\Delta u_{1} \ldots \Delta u_{s}\right) \int_{G} G P\left(G \mid u_{1 j}, \ldots, u_{s_{h}}\right) d G
$$

in which the factor multiplying the integral is just the probability that the $a^{\text {th }}$ cell in the function space is occupied.

By a similar procedure we obtain for the average on the right side of Eq. 58,

$$
\overline{\mathrm{G}(\mathrm{t}) \mathrm{z}(\mathrm{t}) \Phi(a)} \approx \mathrm{P}\left(\mathrm{u}_{1_{j}}, \ldots, \mathrm{u}_{\mathrm{s}_{\mathrm{h}}}\right)\left(\Delta \mathrm{u}_{1} \ldots \Delta \mathrm{u}_{\mathrm{s}}\right) \int_{\mathrm{G}} \int_{\mathrm{z}} \mathrm{G} z \mathrm{P}\left(\mathrm{zG} \mid \mathrm{u}_{1_{j}}, \ldots, \mathrm{u}_{\mathrm{s}_{\mathrm{h}}}\right) \mathrm{dG} \mathrm{dz}
$$

Using Eqs. 71 and 72 in Eq. 58, we obtain

$$
A_{\alpha} \approx \frac{\int_{G} \int_{z} G z P\left(z G \mid u_{1_{j}}, \ldots, u_{s_{h}}\right) d G d z}{\int_{G} G P\left(G \mid u_{1_{j}}, \ldots, u_{s_{h}}\right) d G}
$$

Recall that $A_{a}$ is just the system output when the $a^{\text {th }}$ cell in the function space is occupied. Thus, as the cells become smaller, the system determined by Eq. 58 approaches the optimum system of Eq. 68.

Let us now remove the restriction on the size of the cells. For simplicity in interpreting the results, we shall let $G=1$. For this value of $G$, the weighted 
mean-square-error criterion reduces to the mean-square-error criterion. Equation 58 becomes

$$
\mathrm{A}_{\alpha} \overline{\Phi(a)}=\overline{\mathrm{z}(\mathrm{t}) \Phi(\alpha)}
$$

Expressing the time averages as ensemble averages, we have for $\overline{\Phi(\alpha)}$

$$
\overline{\Phi(a)}=\int_{u_{1}} \ldots \int_{u_{s}} \Phi(\alpha) P\left(u_{1}, \ldots, u_{s}\right) d u_{1} \ldots d u_{s}
$$

But this is just the probability that the $a^{\text {th }}$ cell is occupied. That is,

$$
\overline{\Phi(a)}=\mathrm{P}\left(a^{\text {th }} \text { cell }\right)
$$

For the time average on the right side of Eq. 74, we have

$$
\overline{z(t) \Phi(a)}=\int_{z} \int_{u_{1}} \ldots \int_{u_{s}} z \Phi(a) P\left(u_{1}, \ldots, u_{s} \mid z\right) P(z) d z d u_{1} \ldots d u_{s}
$$

Integrating over the $u^{\prime} s$, we obtain

$$
\overline{z(t) \Phi(a)}=\int_{z} z P\left(a^{\text {th }} \operatorname{cell} \mid z\right) P(z) d z
$$

in which $\mathrm{P}\left(a^{\text {th }}\right.$ cell $\left.\mid \mathrm{z}\right) \mathrm{dz}$ is the probability that the $a^{\text {th }}$ cell is occupied, given that $\mathrm{z}$ is in the interval $\mathrm{dz}$ about $\mathrm{z}$. It is convenient to rewrite Eq. 78 in the form

$$
\overline{z(t) \Phi(a)}=P\left(a^{\text {th }} \text { cell }\right) \int_{z} z P\left(z \mid a^{\text {th }} \text { cell }\right) d z
$$

Using Eqs. 79 and 76 in Eq. 74, we obtain

$$
A_{a}=\int_{z} z P\left(z \mid a^{t h} \text { cell }\right) d z
$$

That is to say, $A_{a}$ is equal to the conditional mean of the desired output, given that the $a^{\text {th }}$ cell in the input function space is occupied. Therefore the results of the filter theory which were developed in the previous sections can be interpreted (for $G(t)=1$ ) as a procedure that quantizes the function space of the input and assigns an output to each cell that is equal to the conditional mean of the desired output, given that this cell is occupied.

It is interesting to note that although we can interpret this filter theory either on a time-domain basis or on a statistical basis, the former leads directly to associated equipment for the filter determination and synthesis, while the latter just expresses 
a mathematical relationship between the quantities involved in the problem. For example, in the simple case in which $G(t)=1$, Eq. 80 might suggest that we evaluate $P\left(z \mid a^{\text {th }}\right.$ cell $)$ for all cells and for all $z$ and then perform the indicated integration. However, the time-domain approach shows us directly that the convenient quantities to measure are those of Eqs. 75 and 79, which look quite formidable from the statistical point of view. Furthermore, when we introduce $G(t)$, the problem appears considerably more complicated from the statistical point of view but, as we have seen from the time-domain approach, it only involves the addition of a single gate circuit in the experimental apparatus (Fig. 13).

\subsection{OPTIMUM NONLINEAR FILTERS FOR A MAXIMUM PROBABILITY CRITERION}

We shall now discuss a method for determining a nonlinear filter whose output is the most probable value of the desired output, given the past of the input. Such a filter can be determined with a simple modification of the apparatus (Fig. 13) that was used to determine optimum filters for a weighted mean-square-error criterion.

As in the case of the weighted mean-square-error criterion, we let the form of the nonlinear operator be that of Eq. 53. At any instant, the system output is equal to the coefficient of the term that corresponds to the occupied cell in the function space of the past of the input. Hence, for the maximum probability criterion, we must choose each coefficient $A_{a}$ to be equal to the most probable value of the desired output, given that the $a^{\text {th }}$ cell is occupied.

An experimental procedure for determining maximum probability filters is shown in Fig. 16. The desired output $z(t)$ is fed into a level-selector circuit. If the amplitude of $z(t)$ is in the amplitude interval corresponding to the level $z_{j}$, then the output at the $j^{\text {th }}$ terminal of the level selector is unity, otherwise it is zero. The outputs of the level-selector circuit, along with the output of the $\Phi$ generator, are fed into gate circuits. The output of the $j^{\text {th }}$ gate circuit is unity when $z(t)$ is in the amplitude interval

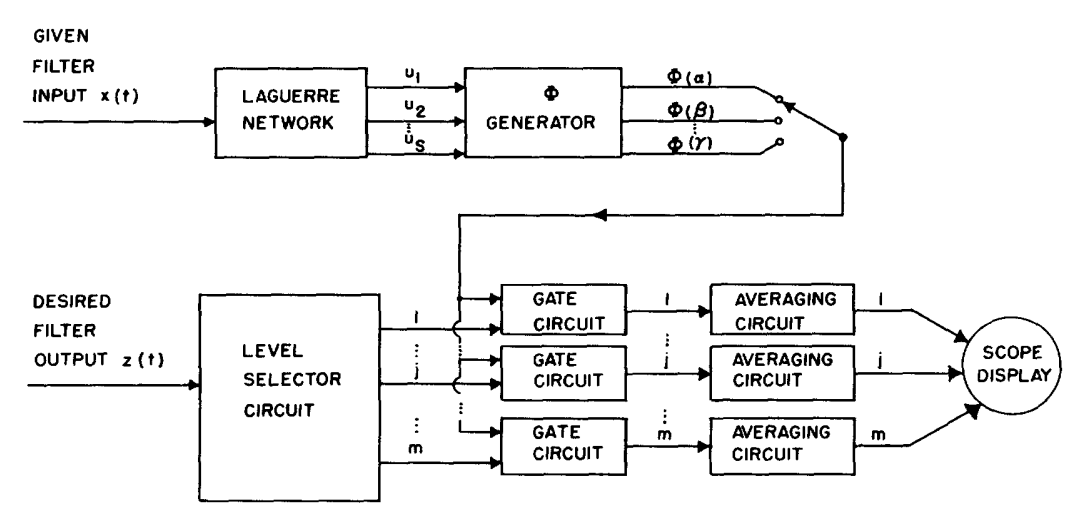

Fig. 16. Experimental procedure for the determination of optimum filters for a maximum probability criterion. 
about $z_{j}$ and, simultaneously, the $a^{\text {th }}$ cell in the function space is occupied. It is zero at all other times. The time average of this output is the probability of the simultaneous occurrence of these two events. We shall write this probability as $\mathrm{P}\left(\mathrm{z}_{j}, a^{\text {th }}\right.$ cell $)$. But

$$
P\left(z_{j}, a^{\text {th }} \text { cell }\right)=P\left(z_{j} \mid a^{\text {th }} \text { cell }\right) P\left(a^{\text {th }} \text { cell }\right)
$$

so that the output of the $j^{\text {th }}$ averaging circuit is proportional to $P\left(z_{j} \mid a^{\text {th }}\right.$ cell $)$. If terminal $\mathrm{k}$ has the largest output of all the $\mathrm{m}$ terminals, then $\mathrm{z}_{\mathrm{k}}$ is the most probable value of $z(t)$, given that the $a^{\text {th }}$ cell is occupied. [It is, of course, assumed that $m$ is large enough so that the amplitude interval associated with $z_{j}$ is very small compared with the maximum amplitude of $z(t)]$. Hence the optimum $A_{a}$ for the maximum probability criterion is equal to $z_{k}$.

For convenience in rapidly determining which output of the averaging circuits is largest, the outputs can be displayed on an oscilloscope as indicated in Fig. 16. Once the $A^{\prime}$ 's are determined, the filter can be synthesized in the standard form shown in Fig. 14.

\subsection{IMPROVING THE PERFORMANCE OF A GIVEN FILTER}

As we increase the complexity of the filter (i.e., we use more Laguerre coefficients to characterize the past and more gate functions for each coefficient) the number of $\mathrm{A}_{a}$ that are necessary to specify the filter grows very rapidly. In particular, if we use $s$ Laguerre coefficients and $n$ gate functions for each coefficient, we have $n^{\mathbf{S}} A_{a}$ to evaluate. After evaluating a large number of $\mathrm{A}_{a}$, we should like to have some guarantee that our filter would perform at least as well as, say, a linear filter or a simple nonlinear filter that can be designed with less effort. Methods of obtaining this guarantee will now be described.

Let us, first of all, prove the existence of a property of our class of filters which will be used in one of the methods. We want to show that the class of filters employing $s$ Laguerre coefficients includes the class of filters that only uses any one of the $s$ Laguerre coefficients. Since we can always renumber the Laguerre coefficients, it is sufficient to prove that the s-coefficient class includes the class that uses only the first Laguerre coefficient $u_{1}$. The series representation for the general system of this one coefficient class is

$$
y(t)=\sum_{i=1}^{n} a_{i} \phi_{i}\left(u_{1}\right)
$$

We now make use of the fact that the sum of the $n$ gate functions of any one coefficient is unity, in order to express the series representation (Eq. 82) in the form 


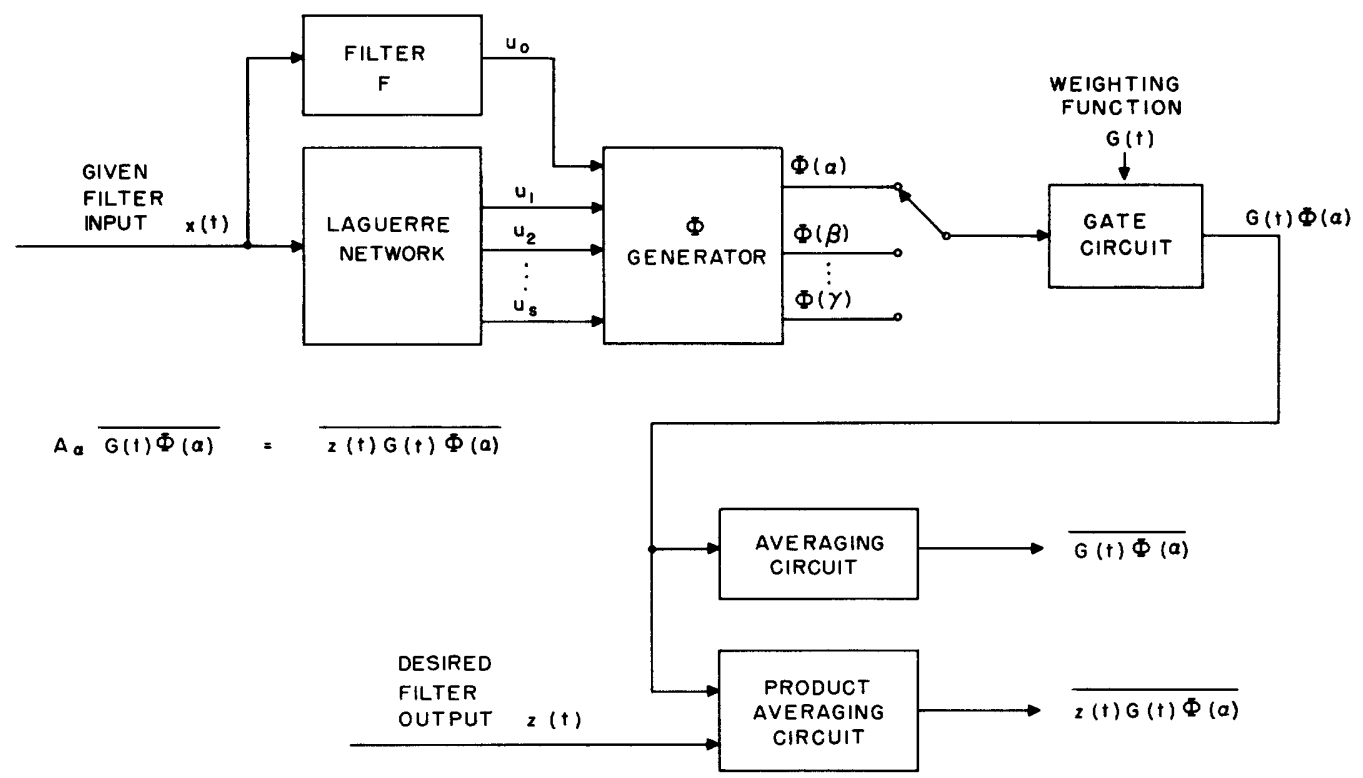

Fig. 17. Augmenting the Laguerre coefficients with the output of a given filter $F$ to determine a filter with a better performance than that of $\mathbf{F}$.

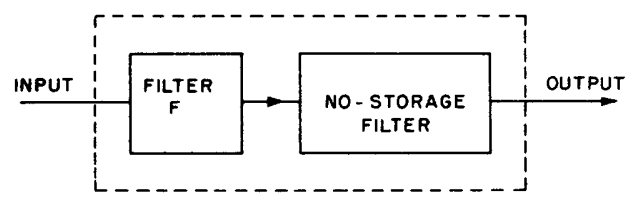

Fig. 18. The class of filters that consists of a given filter F cascaded with a no-storage filter.

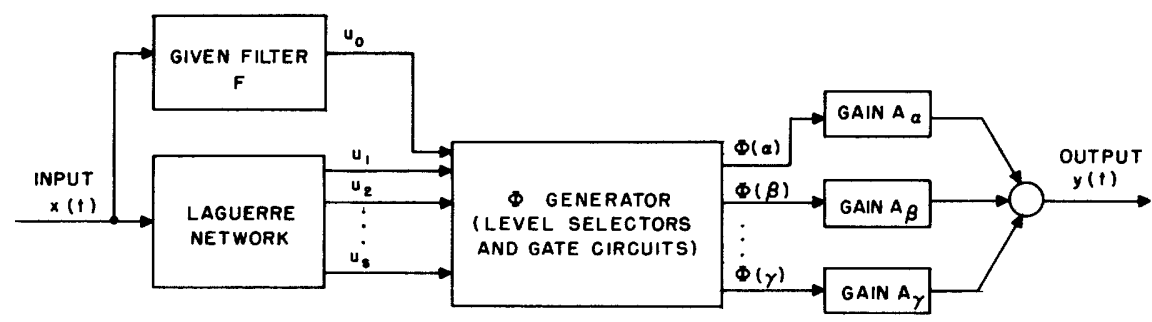

Fig. 19. Synthesis of the filter with coefficients determined in accordance with Fig. 17. 


$$
y(t)=\sum_{i=1}^{n} a_{i} \phi_{i}\left(u_{1}\right)\left[\sum_{j=1}^{n} \phi_{j}\left(u_{2}\right)\right]\left[\sum_{k=1}^{n} \phi_{k}\left(u_{3}\right)\right] \cdots\left[\sum_{h=1}^{n} \phi_{h}\left(u_{s}\right)\right]
$$

which is recognized to be a special case of the expansion (Eq. 51) for the general s-coefficient system. In a similar way, it can be shown that the class of filters using $s$ Laguerre coefficients includes all classes having less than $s$ coefficients. Note that this property is independent of the nature of the $u$ 's; they may be Laguerre coefficients of the past of $x(t)$ or they may be obtained from $x(t)$ by any linear or nonlinear operation.

We now make use of this property to determine a filter whose performance is equal or superior, with respect to a weighted mean-square-error criterion, to a given filter F. F may be linear or nonlinear. We augment the Laguerre coefficients with the output, $u_{0}$, of the given filter $F$, as shown in Fig. 17. Then, by the property demonstrated above, the filter whose output is expressible as

$$
y(t)=\sum_{i=1}^{n} a_{i} \phi_{i}\left(u_{o}\right)
$$

is a member of the class of filters which has $s$ Laguerre coefficients augmented by $u_{o}$. If the number $\mathrm{n}$ of gate functions associated with the variable $u_{0}$ is sufficiently large, then, to any degree of approximation, Eq. 84 represents the class of filters shown in Fig. 18 - the class which consists of $F$ cascaded with a no-storage filter. Since the transfer characteristic of the no-storage system can be linear, the class of filters shown in Fig. 18 certainly contains the filter F. Hence the filter that is determined by the procedure indicated in Fig. 17, for any s, performs at least as well as the given filter $F$ and, in fact, at least as well as $F$ cascaded with any no-storage filter.

After the $\mathrm{A}_{\alpha}$ of the desired filter have been determined, as indicated in Fig. 17, the filter synthesis is accomplished as shown in Fig. 19.

We now turn to another method of determining filters to improve the performance of given filters. Let the output of the given filter $F$ be $u_{0}(t)$ when its input is $x(t)$. Our object is to improve (with respect to a weighted mean-square-error criterion) the performance of $\mathrm{F}$ by paralleling it with a filter which will be determined. The $A_{a}$ of the desired filter are those that minimize the error

$$
\mathscr{E}=\lim _{T \rightarrow \infty} \frac{1}{2 T} \int_{-T}^{T} G(t)\left\{z(t)-\left[u_{o}(t)+\sum_{a} A_{a} \Phi(a)\right]\right\}^{2} d t
$$

This expression is seen to be equivalent to

$$
\mathscr{E}=\lim _{T \rightarrow \infty} \frac{1}{2 T} \int_{-T}^{T} G(t)\left\{\left[z(t)-u_{0}(t)\right]-\sum_{a} A_{a} \Phi(a)\right\}^{2} d t
$$




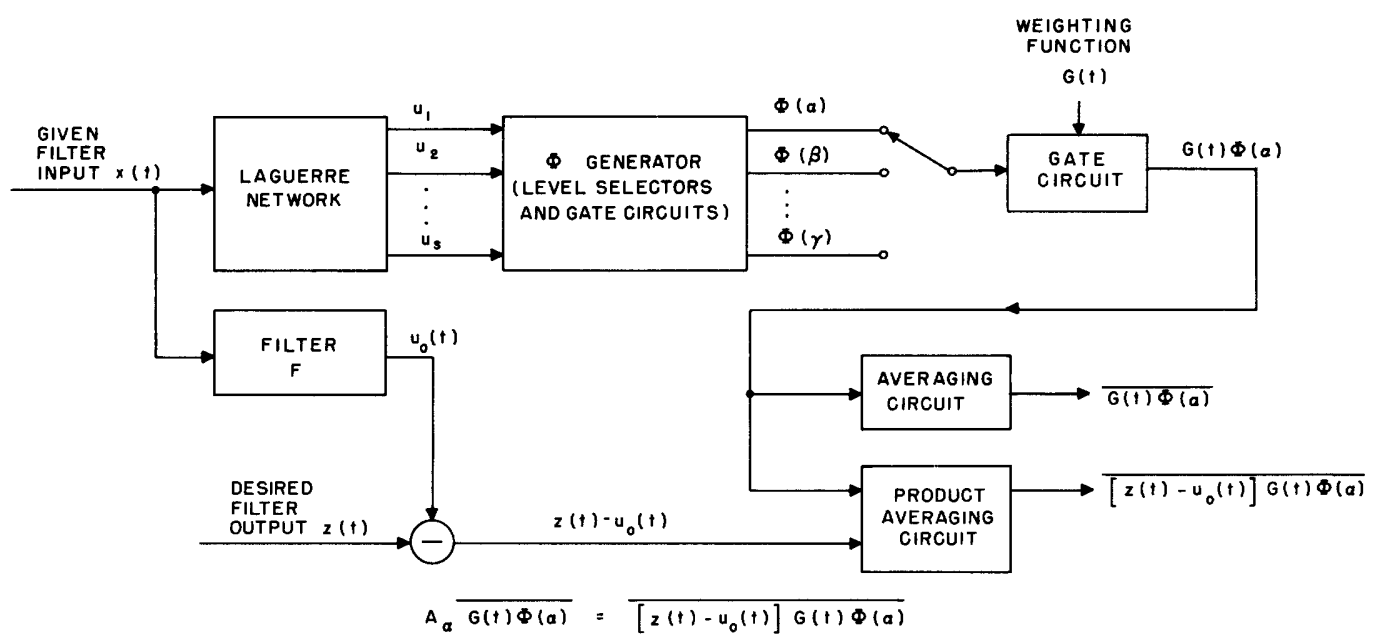

Fig. 20. Apparatus for the determination of a filter to improve the performance of $F$ by a parallel connection.

Comparing Eq. 86 with Eq. 54, we see that the optimum $\mathrm{A}_{a}$ are determined by an experimental procedure like that indicated in Fig. 13, with $z(t)$ replaced by $z(t)-u_{0}(t)$. The latter quantity is easily obtained by applying $x(t)$ to the given filter $F$ and subtracting the output of $F$ from $z(t)$, as shown in Fig. 20. The parallel combination of $F$ and the filter, determined as we have just described, will always perform at least as well as $F$, since that filter which has no transmission from input to output is a member of the class of filters considered in our theory. In other words, the solution in which all the $A_{a}$ are equal to zero is a possible solution of Eqs. 84 and 85 .

The second method described for improving the performance of given filters offers the advantage of not having gate functions associated with the output time function of the given filter; therefore, improvements can be made on the performance of $F$ by very simple systems that involve as few as one Laguerre coefficient and thus have a relatively small number of $A_{a}$ to evaluate. The first method does require a gate-function expansion of the output of the given filter $F$ but it has the advantage of ensuring that the performance of the resultant filter will always be at least as good as the performance of $\mathrm{F}$ cascaded with any no-storage system. In either method, the resultant over-all filter approaches the most general filter (of the class considered in this theory) as the number of Laguerre coefficients and gate functions is increased.

Still another design procedure involves the determination of that filter which, when cascaded with $F$ (with $F$ as the first member of the combination), yields an over-all filter with a performance superior to that of $F$ alone. In order to ensure that the resultant over-all filter performs at least as well as F, we could augment the Laguerre coefficients of the cascaded filter by a variable $u_{0}$ that is equal to the input of the Laguerre network. While this procedure gives a filter that is at least as good as F, we have no assurance that as the number of Laguerre coefficients and Hermite functions is increased the over-all filter will approach the most general filter. 


\subsection{MULTIPLE NONLINEAR PREDICTION}

The problem of multiple prediction is that of predicting a time series from a knowledge of related time series. An example, cited by Wiener, is the prediction of weather at one location from a knowledge of the past of the weather at that and other surrounding locations.

The filter theory developed in the preceding sections is easily extended to the problem of multiple prediction. Let $z(t+a)$ be the function that we desire to predict and let $x_{1}(t)$ through $x_{p}(t)$ be the input functions on whose past we operate to form our prediction. The set of functions $x_{1}(t)$ through $x_{p}(t)$ may, indeed, include $z(t)$. We shall characterize the past of each input by a set of Laguerre coefficients. Let ${ }_{j} u_{1} j_{2} \cdots_{j}{ }_{j} u_{s}$ be the Laguerre coefficients of $x_{j}(t)$. Now let us think of a function space that encompasses the past of all $\mathrm{p}$ of the input time functions. That is, the basis of this space is formed by the Laguerre functions associated with each input. A point in this space then represents the past of all the inputs and thus the multiple prediction problem is just the problem of mapping this space onto a line (corresponding to the amplitude of the system output) in a manner that is optimum with respect to some error criterion. But this is the same problem encountered in the single-input filter problem and we recognize that the only difference between the single - and multiple-input problems is in the number of dimensions of the function space. The solution of the multiple prediction problem directly parallels that of the filter problem given in section 3.4.

We let $y(t)$ be a gate-function expansion of the Laguerre coefficients of all the inputs $x_{1}(t) \ldots x_{p}(t)$. The expansion is

$$
\begin{aligned}
y(t)= & \sum_{i_{1}} \sum_{j_{1}} \ldots \sum_{h_{1}} \ldots \sum_{i_{p}} \sum_{j_{p}} \ldots \sum_{h_{p}} a_{i_{1}, j_{1}} \ldots, h_{1}, \ldots, i_{p}, j_{p}, \ldots, h_{p} \\
& \times \phi_{i_{1}}\left(u_{1}\right) \phi_{j_{1}}\left(1 u_{2}\right) \ldots \phi_{h_{1}}\left(u_{s}\right) \ldots \phi_{i_{p}}\left(u_{1}\right) \phi_{j_{p}}\left(u_{2} u_{2} \ldots \phi_{h_{p}}\left(u_{s}\right)\right.
\end{aligned}
$$

If we associate an $\Omega(a)$ with each product of $\phi^{\prime} \mathrm{S}$ in Eq. 87 and let $\mathrm{A}_{a}$ be the corresponding coefficient $a_{i_{1}}, j_{1}, \ldots, h_{1}, \ldots, i_{p}, j_{p}, \ldots, h_{p}$, Eq. 87 takes the simplified form

$$
y(t)=\sum_{a} A_{a} \Omega(a)
$$

Just as in the filter problem (sec. 3.4), we adopt a weighted mean-square-error criterion and minimize the error

$$
\mathscr{E}=\lim _{T \rightarrow \infty} \frac{1}{2 T} \int_{-T}^{T} G(t)\left\{z(t+a)-\sum_{a} A_{\alpha} \Omega(a)\right\}^{2} d t
$$




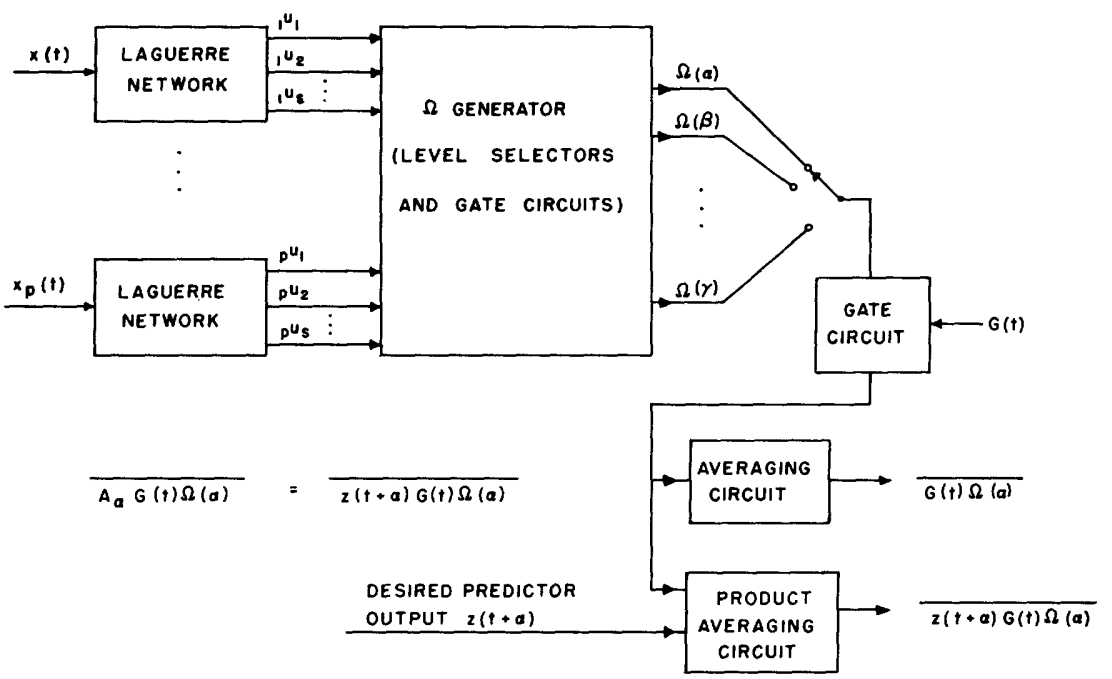

Fig. 21. Experimental procedure for the determination of the optimum multiple predictor.

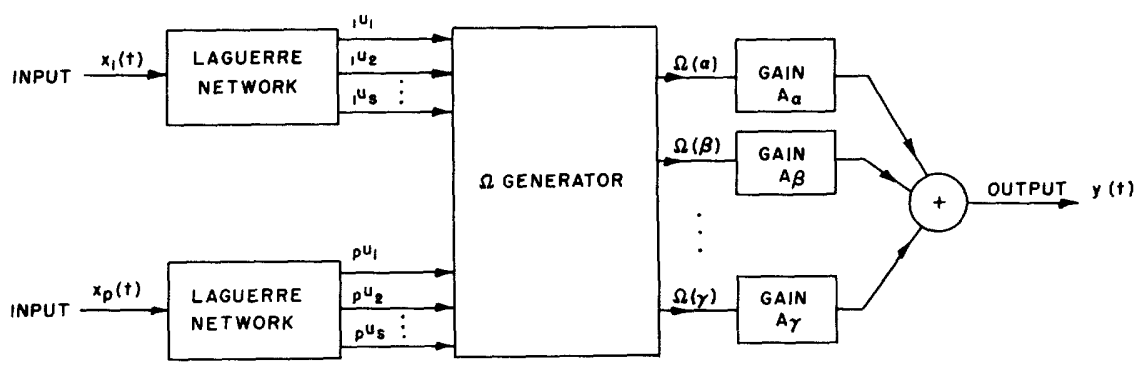

Fig. 22. Synthesis of the optimum multiple predictor.

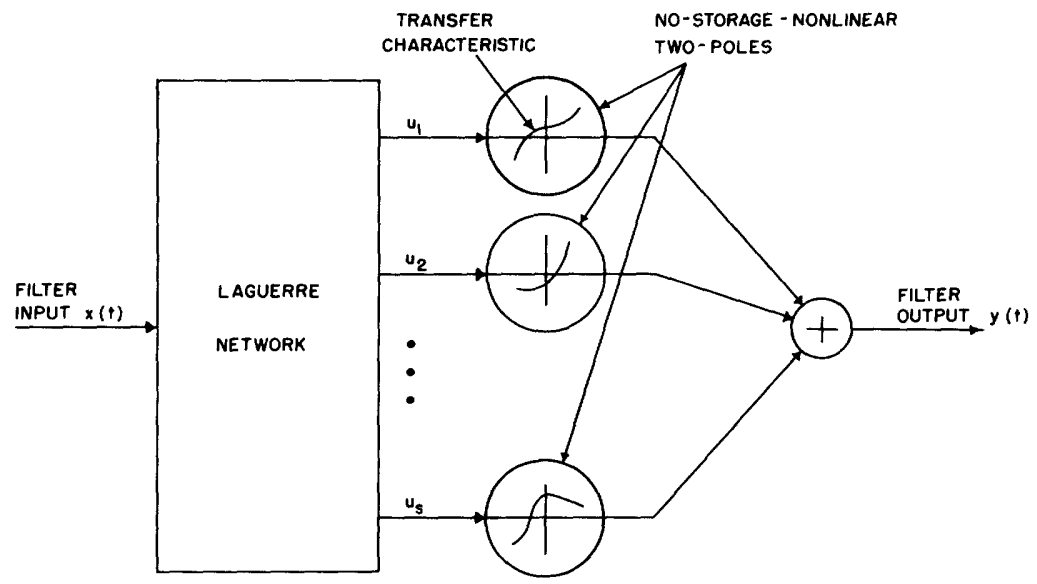

Fig. 23. The class of nonlinear systems that has no cross products of the Laguerre coefficients. 
This equation is seen to have the same form as Eq. 54 for the filter problem. The solution for the optimum $A_{a}$ proceeds exactly as in section 3.4, with the result that

$$
A_{a} \overline{G(t) \Omega(a)}=\overline{z(t+a) G(t) \Omega(a)}
$$

The apparatus for experimentally determining the $\mathrm{A}_{a}$ according to this equation is shown in Fig. 21. After the coefficients have been determined, the predictor can be synthesized in accordance with Eq. 88, as shown in Fig. 22.

\section{LINEAR AND SIMPLE NONLINEAR SYSTEMS}

\subsection{A TEST FOR THE COEFFICIENTS}

Once the coefficients for an optimum filter have been determined, the filter can, as we have seen, be synthesized formally, as shown in Fig. 14. From the standpoint of apparatus, much simpler synthesis procedures exist, however, if the filter is linear or belongs to a particular subclass of nonlinear systems. Hence it is desirable to have a means of detecting linear and simple nonlinear systems directly from a knowledge of their characterizing coefficients. We shall develop a simple procedure for testing the coefficients $\left(\mathrm{A}_{\alpha}\right)$ that detects such systems and directly yields a convenient synthesis of them.

The class of simple nonlinear systems that we shall consider is shown in Fig. 23. It consists of a Laguerre network and no-storage nonlinear two-pole circuits (no-storage nonlinear systems with one input and one output terminal). Each output of the Laguerre network is fed into one no-storage two-pole circuit and the outputs of these circuits are added to form the system output $y(t)$. In this class of systems the nonlinear circuits introduce no cross talk among the Laguerre coefficients (i.e., no cross products of Laguerre coefficients are introduced). This class of systems is clearly a subclass of the general class considered in Section III. When the transfer characteristics of all the two-pole circuits are straight lines, the system is linear. In particular, it is an $s^{\text {th }}$ order Laguerre network in which the gain factors associated with the Laguerre network outputs are equal to the slopes of the respective linear two-pole transfer characteristics.

The synthesis of nonlinear systems belonging to this subclass is relatively simple. The nonlinear two-pole circuits can be synthesized by piecewise-linear approximations with the use of diodes and resistors. If the system is linear, the synthesis takes the form of a Laguerre network whose outputs are properly amplified or attenuated before being added to form the system output. In this linear case it may be desirable to measure the transfer function of the optimum system and then use available synthesis techniques to obtain alternate realizations of this transfer function by using linear passive circuits.

We now investigate the determination, from the characterizing coefficients $A_{a}$, of a nonlinear system, if the system belongs to the class shown in Fig. 23. If it does, we 
shall investigate the determination of the transfer characteristics of the nonlinear two-pole circuits.

From the point of view of function space, the subclass of nonlinear systems shown in Fig. 23 consists of all those systems for which the system output corresponding to each cell in function space is equal to the sum of the outputs corresponding to the coordinates (the Laguerre coefficients) of the cell. That is, since there is no cross talk, each coordinate contributes to the output an amount that is independent of the other coordinates and thus the system output corresponding to any cell is the sum of the outputs corresponding to the coordinates of the cell. (We speak here of cells rather than points in function space because we represent our nonlinear system by a gate-function expansion that quantizes the function space. In this connection we should also realize that we obtain a stepwise approximation to the two-pole characteristics of Fig. 23 rather than the continuous curves.) Hence Eq. 51, which represents a general gatefunction expansion of the Laguerre coefficients, takes on the form

$$
y(t)=\sum_{i=1}^{n} b_{i 1} \phi_{i}\left(u_{1}\right)+\sum_{j=1}^{n} b_{j 2} \phi_{j}\left(u_{2}\right)+\ldots+\sum_{h=1}^{n} b_{h s} \phi_{h}\left(u_{s}\right)
$$

for the subclass of systems of Fig. 23. Each sum in the equation yields the transfer characteristic of the nonlinear two-pole circuit that is associated with the Laguerre coefficient indicated in that sum. Compare this expansion with that of Eq. 51.

In the experimental procedure described in Section III for finding optimum filters (Fig. 13) we determine the $A_{a}$ or equivalently the $a_{i, j}, \ldots, h$ of the filter. If and only if these a's are such that Eq. 51 can be expressed in the form of Eq. 91, the system can be synthesized in accordance with Eq. 91 in the form shown in Fig. 23. Let us see how the a's must be related to the b's if these two equations are to be equivalent. By "equivalent" we mean that the two equations yield the same value for every cell in function space; hence we must have

$$
a_{i, j}, \ldots, h=b_{i 1}+b_{j 2}+\ldots+b_{h s} \quad \text { for all } i, j, \ldots, h
$$

This relation represents a set of $n^{S}$ simultaneous equations which the a's must satisfy. We shall now develop a simple way of finding out whether or not, for any given set of $a^{\prime}$ 's, this set of equations is satisfied.

It is convenient to establish an order for the evaluation of the a's (and thus the $A_{a}$ ). It is assumed, henceforth, that these coefficients are evaluated as follows. The first coefficient we evaluate is that for which $i=j=\ldots=h=1$. The next $n-1$ coefficients are obtained by letting $h$ run from 2 to $n$ while all other indices are held equal to unity. To obtain the $(n+1)^{\text {th }}$ coefficient, we set the index preceding $h$ to 2 and let all other indices be unity. The following $n-1$ coefficients are obtained by again letting $h$ range from 2 to $n$. We continue this procedure until the index $i$ has gone through all its n-values, at which point all the a's will have been evaluated. This order of evaluating 
the coefficients is best illustrated by a simple example.

EXAMPLE 4. Consider the coefficients (a's) of a nonlinear system that has three Laguerre coefficients and two gate functions for each coefficient. That is, $s=3$ and $\mathrm{n}=2$. There are $\mathrm{n}^{\mathrm{S}}=8$ coefficients $\mathrm{a}_{\mathrm{i}, \mathrm{j}, \mathrm{k}}$ to evaluate. According to the above procedure, these coefficients are evaluated in the following order:
1. $a_{111}$
5. $a_{211}$
2. $\mathrm{a}_{112}$
6. $a_{212}$
3. $a_{121}$
7. $a_{221}$
4. $\mathrm{a}_{122}$
8. $\mathbf{a}_{222}$

A study of the order in this simple example is sufficient to establish the order of evaluating the $a^{\prime} s$ for any $s$ and $n$.

Now think of the coefficients $a_{i, j}, \ldots, h$, arranged in the order of evaluation, as components of a vector $A$, and the corresponding coefficients $b_{11}, b_{21}, \ldots, b_{n 1}, b_{12}, b_{22}, \ldots, b_{n 2}, \ldots, b_{1 s}, b_{2 s}, \ldots, b_{n s}$, arranged as shown, as components of a vector B. Then the set of equations represented by Eq. 92 can be written in matrix form as follows:

$$
\mathrm{A}]=[\mathrm{M}] \mathrm{B}]
$$

where $[M]$ is the matrix that operates on the vector $B$ to give the vector $A$. Let us determine the form of the matrix $[\mathrm{M}]$. In order to illustrate the form of this matrix we shall consider a nonlinear system for which $\mathrm{s}=\mathrm{n}=3$ and for which Eq. 92 is assumed to hold. From the results of example 4 the form of [M] can be visualized for any $s$ and $n$. The equations indicated in Eq. 92 are given, for this example, in Table I. The b's are written at the top of the columns so that the form of the matrix $[\mathrm{M}]$ is evident. The actual equations are obtained by dropping the b's down beside the unity coefficients. (All the blank spaces in the matrix represent zero matrix coefficients.) Thus the first equation reads

$$
a_{111}=b_{11}+b_{12}+b_{13}
$$

We see that the matrix [M] is composed entirely of zeros and ones. We also note the regular pattern of the unity coefficients. A study of this pattern will enable the reader to visualize its form for any $s$ and $n$.

We now state a test for the a's that enables us to determine directly whether or not the set of equations (Eq. 92) or, equivalently, Eq. 93 is satisfied. The test was developed from a study of $[M]$ and its validity can be checked (for $s=n=3$ ) by analyzing it in terms of the matrix of Table I.

1. Starting with $a_{1}, 1, \ldots, 1$, plot the a's (consecutively in the order in which they are evaluated) in groups of $\mathrm{n}$ at unit intervals along a linear scale. That is, form a set of $n^{(s-1)}$ graphs, each of which contains $n a^{\prime}$ s plotted at equal intervals along a 


\begin{tabular}{|c|c|c|c|c|c|c|c|c|c|c|}
\hline & & $b_{11}$ & $\mathrm{~b}_{21}$ & $b_{31}$ & $b_{12}$ & $b_{22}$ & $b_{32}$ & $b_{13}$ & $\mathrm{~b}_{23}$ & $\mathrm{~b}_{33}$ \\
\hline$a_{111}$ & $=$ & 1 & & & 1 & & & I & & \\
\hline$a_{112}$ & $=$ & 1 & & & 1 & & & & 1 & \\
\hline$a_{113}$ & $=$ & 1 & & & 1 & & & & & 1 \\
\hline$a_{121}$ & $=$ & 1 & & & & 1 & & 1 & & \\
\hline$a_{122}$ & $=$ & 1 & & & & 1 & & & 1 & \\
\hline$a_{123}$ & $=$ & 1 & & & & 1 & & & & 1 \\
\hline$a_{131}$ & $=$ & 1 & & & & & 1 & 1 & & \\
\hline$a_{132}$ & $=$ & 1 & & & & & 1 & & 1 & \\
\hline$a_{133}$ & $=$ & 1 & & & & & 1 & & & 1 \\
\hline$a_{211}$ & $=$ & & 1 & & 1 & & & 1 & & \\
\hline$a_{212}$ & $=$ & & 1 & & 1 & & & & 1 & \\
\hline$a_{213}$ & $=$ & & 1 & & 1 & & & & & 1 \\
\hline$a_{221}$ & $=$ & & 1 & & & 1 & & 1 & & \\
\hline$a_{222}$ & $=$ & & 1 & & & 1 & & & 1 & \\
\hline$a_{223}$ & $=$ & & 1 & & & 1 & & & & 1 \\
\hline$a_{231}$ & $=$ & & 1 & & & & I & 1 & & \\
\hline$a_{232}$ & $=$ & & 1 & & & & 1 & & 1 & \\
\hline$a_{233}$ & $=$ & & 1 & & & & 1 & & & 1 \\
\hline$a_{311}$ & $=$ & & & 1 & 1 & & & 1 & & \\
\hline$a_{312}$ & $=$ & & & 1 & 1 & & & & 1 & \\
\hline$a_{313}$ & $=$ & & & 1 & 1 & & & & & 1 \\
\hline$a_{321}$ & $=$ & & & 1 & & 1 & & 1 & & \\
\hline$a_{322}$ & $=$ & & & 1 & & 1 & & & 1 & \\
\hline$a_{323}$ & $=$ & & & 1 & & 1 & & & & 1 \\
\hline$a_{331}$ & $=$ & & & 1 & & & 1 & 1 & & \\
\hline$a_{332}$ & $=$ & & & 1 & & & 1 & & 1 & \\
\hline$a_{333}$ & $=$ & & & 1 & & & 1 & & & 1 \\
\hline
\end{tabular}

line. Call this set of graphs set 1 .

2. Take the first "a" of each graph above, starting with $a_{1,1, \ldots, 1}$, and plot these consecutively in groups of $\mathbf{n}$ at unit intervals on a linear scale. Call this set of graphs set 2 .

3. Repeat the procedure of step 2 until $s$ sets of graphs are obtained. The $s^{\text {th }}$ set will consist of only one graph.

From a study of the general form of $[M]$ it can be seen that if, for a given set of a's, there exists a set of $b^{\prime} s$ that are such that Eq. 93 is satisfied, then all of the graphs of these a's within each set of graphs will be identical, except for a possible vertical translation. Furthermore, the converse holds; that is, if all of the graphs of the a's within 
each set of graphs are identical, within a vertical translation, then there exists a set of b's that are such that Eq. 93 is satisfied.

Hence, if there exists a system governed by Eq. 91 that is equivalent to a system governed by Eq. 51 for a given set of a's, then all the graphs of the a's within each set of graphs must be the same, except for a possible vertical translation. And, conversely, if we apply the above test to the a's of a system and find that all the graphs within each set are the same, within vertical translation, we know that the system that has these a's can be synthesized in the form shown in Fig. 23.

\section{2 SYNTHESIS PROCEDURE}

In this section we assume that the a's have been tested as described above and that they correspond to a system of the type shown in Fig. 23. We are now concerned with the synthesis of this system. In particular, we want to find the transfer characteristics of the no-storage two-pole circuits. One way to do this is to solve Eq. 93 for the b's, which, by Eq. 91, directly determine the no-storage transfer characteristics. As a consequence of the special form of $[\mathrm{M}]$, this solution is readily accomplished. However, a simpler method of synthesizing the desired system makes direct use of the graphs that are drawn when the a's are tested. Referring to the previous section, let us examine, relative to the a's in Table I, the sets of graphs defined in the test procedure. From inspection of Table $I$ it is readily seen that all the graphs of set 1 are identical to (except for a possible vertical translation) the graph that would be obtained if $b_{13}, b_{23}$, and $b_{33}$ were plotted, in this order, at unit intervals on the same linear scale. Similarly, it is seen that the graphs of set 2 are the same (within a vertical translation) as the graph that would be obtained by plotting $b_{12}, b_{22}$, and $b_{32}$. And, finally, the graphs of set 3 correspond, in a similar manner, to the graph that would be obtained by plotting $b_{11}, b_{21}$, and $b_{31}$. But notice (Eq. 91 for $n=s=3$ ) that $b_{13}, b_{23}$, and $b_{33}$ are just the heights of the steps in the stepwise approximation to the two-pole characteristic associated with $u_{3}$. Similarly, $b_{12}, b_{22}$, and $b_{32}$ determine the transfer characteristic of the nonlinear two-pole circuit whose input is $u_{2}$. And, finally, $b_{11}, b_{21}$, and $b_{31}$ determine the two-pole characteristic associated with $u_{1}$. Hence the graphs that are made when the a's are tested directly determine the desired two-pole transfer characteristics, within a vertical translation. In the synthesized nonlinear system (Fig. 23) the vertical displacement of the two-pole transfer characteristics affects only the dc level of the output. When this level is of interest it can be re-established by a series battery at the system output, as will be shown in examples that follow.

Now we can readily generalize to the case of arbitrary $s$ and $n$. Except for a vertical translation, the graph of set 1 determines the transfer characteristic of the two-pole circuit associated with $u_{s}$. The graph of set 2 determines the transfer characteristic of the two-pole circuit associated with $u_{s-1}$, and so on, down to the graph of set $s$, which determines the transfer characteristic of the two-pole circuit associated with $u_{1}$. 


$\begin{array}{lll}a_{111}=1 & a_{211}=0 & a_{311}=2 \\ a_{112}=4 & a_{212}=3 & a_{312}=5 \\ a_{113}=3 & a_{213}=2 & a_{313}=4 \\ a_{121}=2 & a_{221}=1 & a_{321}=3 \\ a_{122}=5 & a_{222}=4 & a_{322}=6 \\ a_{123}=4 & a_{223}=3 & a_{323}=5 \\ a_{131}=5 & a_{231}=4 & a_{331}=6 \\ a_{132}=8 & a_{232}=7 & a_{332}=9 \\ a_{133}=7 & a_{233}=6 & a_{333}=8\end{array}$

EXAMPLE 5. Suppose that we have determined the coefficients of an optimum filter of the class $s=n=3$ and that they have the values given above.

We shall test these coefficients to see if the corresponding system can be synthesized in the form shown in Fig. 23. The coefficients, plotted according to the test procedure described in section 4.1, are shown in Fig. 24. In order to make the form of each graph stand out, consecutive points corresponding to the a's have been joined by straight lines. We see that all the graphs within each set of graphs are identical, within a vertical translation. Hence the system can be synthesized in the form shown in Fig. 23.

The synthesized system is shown in Fig. 25. The transfer characteristics of the no-storage two-pole circuits are obtained directly from the graphs of Fig. 24 . The graph of set 3 is the transfer characteristic of the two-pole circuit associated with $u_{1}$. That is, $a_{111}$ is the coefficient of the first gate function $\phi_{1}\left(u_{1}\right), a_{211}$ is the coefficient of $\phi_{2}\left(u_{1}\right)$, and $a_{311}$ is the coefficient of $\phi_{3}\left(u_{1}\right)$. Similarly, any graph in set 2 can be taken as the transfer characteristic associated with $u_{2}$. And finally, any graph in set 1 can be taken as the transfer characteristic associated with $u_{3}$. As we found earlier, the vertical translation of these transfer characteristics is unimportant, since it can be compensated for by a battery in series with the system output, as shown in Fig. 25. To find the value of the battery, we simply find the output of the system of Fig. 25 for any cell, say cell $1,1,1$, and choose the battery so that this output is equal to that given by the a's. That is, for cell $1,1,1$ the system output (Fig. 25) without the battery is 2 volts (the scales for the transfer characteristics are assumed to be in volts). But the system output for this cell should be equal to 1 volt, since $a_{111}=1$. Hence we insert a one-volt battery, with the proper polarity, in series with the output of the system in Fig. 25. Note that, in any system of the form shown in Fig. 23 or Fig. 25, the battery can always be absorbed in the no-storage transfer characteristics by simply translating one or more of them vertically.

In Fig. 24 we arbitrarily drew straight lines between the plots of the a's. This amounts to forming a transfer characteristic by a linear interpolation between the coefficients that specify the characteristic, as shown by the solid lines in Fig. 25. However, as we have seen, a gate-function expansion yields a stepwise approximation to the transfer 


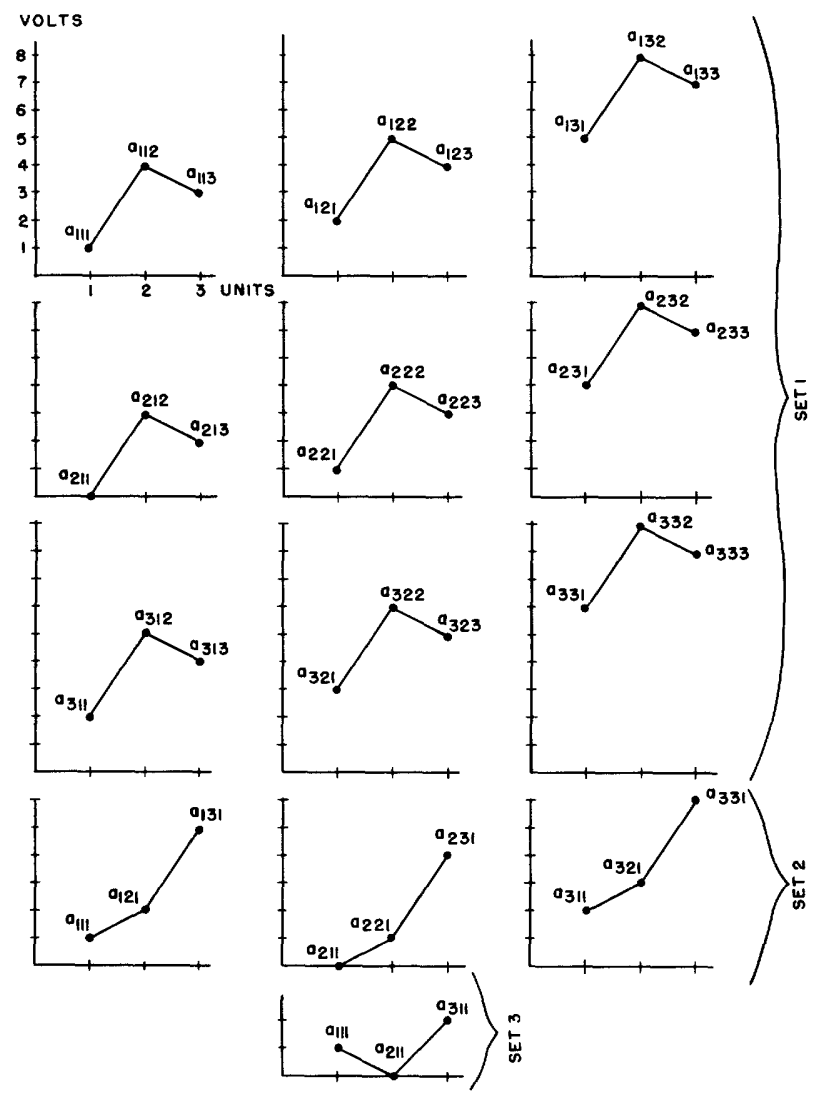

Fig. 24. Graphs of the coefficients in Example 5.

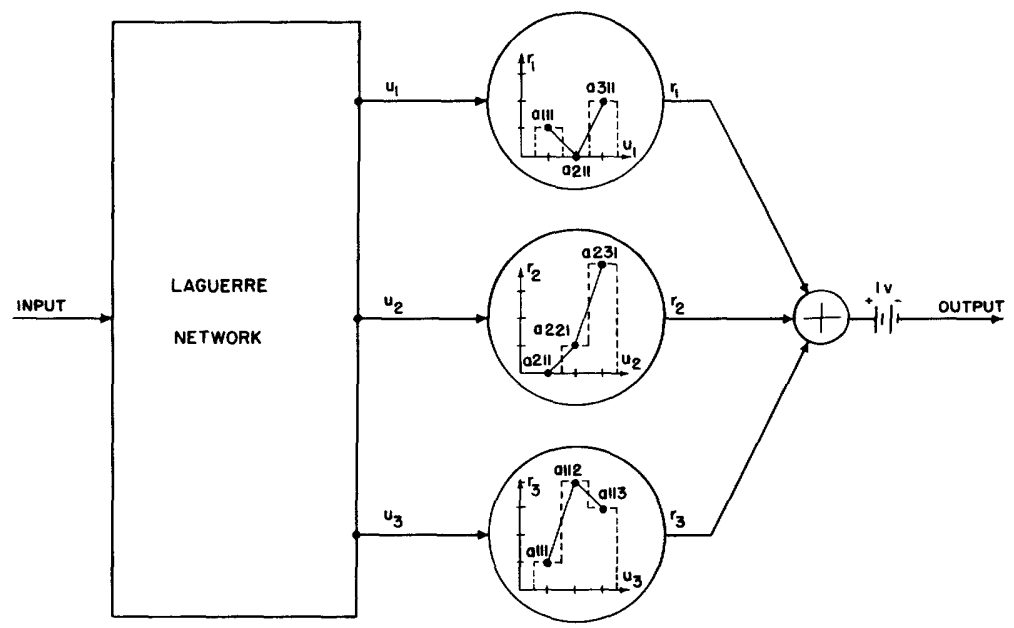

Fig. 25. The nonlinear filter of Example 5 . 


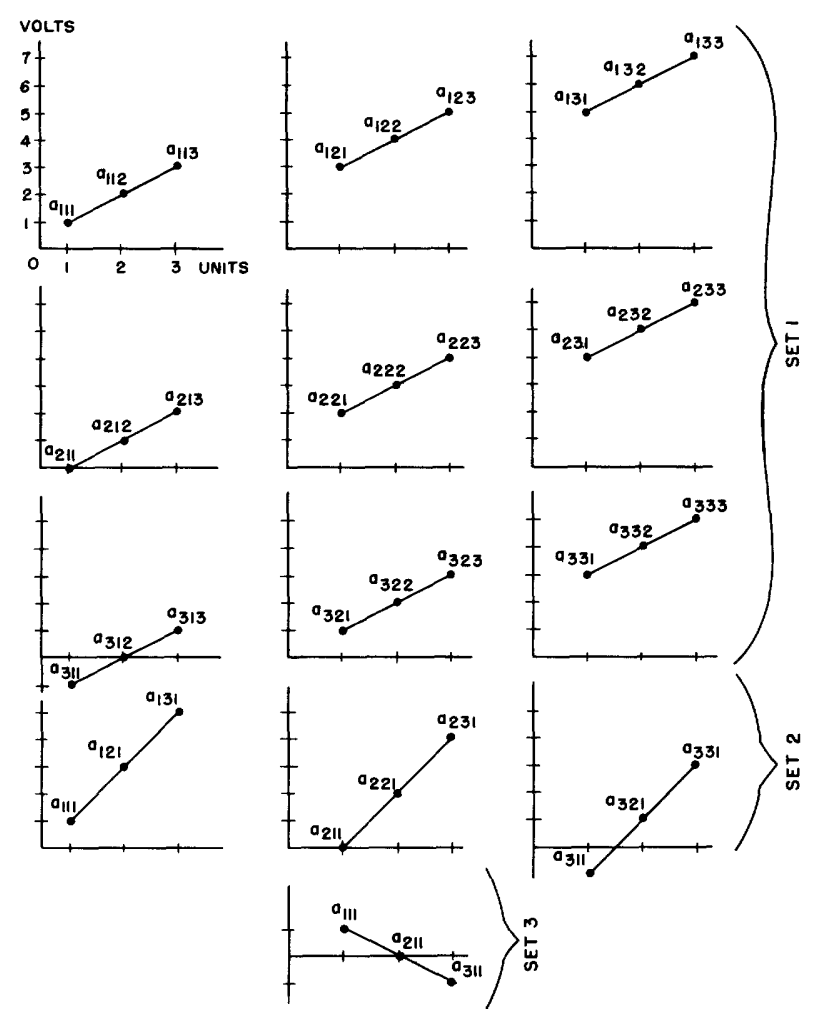

Fig. 26. Graphs of the coefficients in Example 6 .

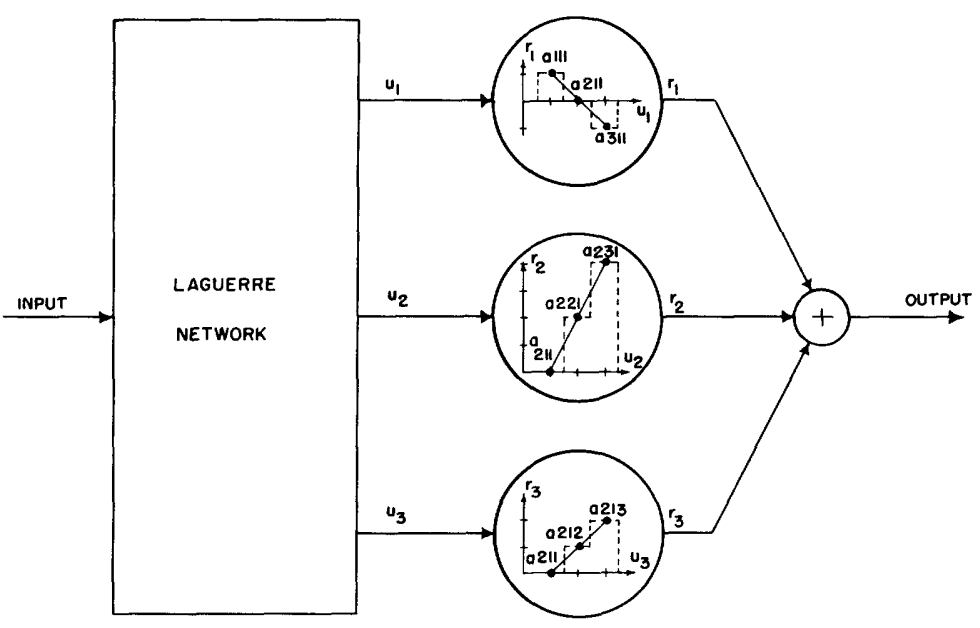

Fig. 27. The filter of Example 6. 
characteristic in which the height of each step is equal to the corresponding coefficient in the expansion. This step curve is shown by dotted lines in Fig. 25.

EXAMPLE 6. Again, we consider a filter of the class $s=n=3$. Let the coefficients for this filter be

$\begin{array}{lll}a_{111}=1 & a_{211}=0 & a_{311}=-1 \\ a_{112}=2 & a_{212}=1 & a_{312}=0 \\ a_{113}=3 & a_{213}=2 & a_{313}=1 \\ a_{121}=3 & a_{221}=2 & a_{321}=1 \\ a_{122}=4 & a_{222}=3 & a_{322}=2 \\ a_{123}=5 & a_{223}=4 & a_{323}=3 \\ a_{131}=5 & a_{231}=4 & a_{331}=3 \\ a_{132}=6 & a_{232}=5 & a_{332}=4 \\ a_{133}=7 & a_{233}=6 & a_{333}=5\end{array}$

These coefficients, plotted according to the test procedure described in section 4.1, are shown in Fig. 26. We see that all the graphs within each set of graphs are identical, within a vertical translation, and, furthermore, we see that they are all linear. Hence the system can be synthesized as shown in Fig. 27. The transfer characteristics of the no-storage two-poles are found directly from the graphs of Fig. 26, as discussed in example 5. The solid lines in the transfer characteristics of Fig. 27 indicate a linear interpolation between the coefficients that specify these characteristics. Since these solid-line transfer characteristics are all linear, they can be replaced by amplifiers whose gains are equal to the slope of the lines.

\subsection{APPROXIMATING FILTERS BY LINEAR AND SIMPLE NONLINEAR FLTERS}

We have seen that, if the graphs of the characterizing coefficients (the a's) of a system satisfy certain conditions, the system can be synthesized in the relatively simple form shown in Fig. 23 and that under certain additional conditions the system is linear and the synthesis even simpler. In practical problems it is unlikely that the system coefficients will exactly satisfy these conditions. However, the relative simplicity of the systems of Fig. 23 makes it worth while for us to determine when a more complicated system can be approximated by one that has this simple form.

The concept of approximating one system by another is meaningful only when we specify a criterion for the approximation and specify the degree of approximation relative to this criterion. In this section we consider the approximation of one system by another from the point of view of two different error criteria.

We first consider the weighted mean-square-error criterion defined by Eq. 54 . Relative to this criterion, we ask how much error is introduced if we change the 
coefficients of the system from their optimum values $A_{a}$ to the values $A_{a}^{\prime}$.

In accordance with Eq. 54, the error for a system whose coefficients are $A_{a}^{\prime}$ is given by

$$
\mathscr{E}=\lim _{T \rightarrow \infty} \frac{1}{2 T} \int_{-T}^{T} G(t)\left\{z(t)-\sum_{a} A_{\alpha}^{\prime} \Phi(a)\right\}^{2} d t
$$

Expanding this equation, we have

$$
\mathscr{E}=\overline{z^{2}(t) G(t)}-\overline{2 G(t) z(t) \sum_{a} A_{a}^{\prime} \Phi(\alpha)}+\overline{G(t) \sum_{a} \sum_{\beta} A_{\alpha}^{\prime} A_{\beta}^{\prime} \Phi(a) \Phi(\beta)}
$$

Taking advantage of the time-domain orthogonality of the $\Phi(a)$, we can rewrite Eq. 96

$$
\mathscr{E}=\overline{z^{2}(t) G(t)}-2 \sum_{a} A_{a}^{\prime} \overline{G(t) z(t) \Phi(a)}+\sum_{a} A_{a}^{\prime 2} \overline{G(t) \Phi(a)}
$$

in which $\Phi^{2}(a)$ has been replaced by its equivalent, $\Phi(a)$.

For the optimum filter coefficients $A_{a}, \mathscr{E}$ takes on its minimum value $\mathscr{E}_{\text {min }}$ given by Eq. 62, as follows

$$
\mathscr{E}_{\min }=\overline{z^{2}(t) G(t)}-\sum_{a} \overline{\frac{G(t) z(t) \Phi(a)^{2}}{\overline{G(t) \Phi(a)}}}
$$

If we use Eq. 58 for the optimum filter coefficients, we can rewrite Eq. 62

$$
\mathscr{E}_{\min }=\overline{z^{2}(t) G(t)}-\sum_{a} A_{a} \overline{G(t) z(t) \Phi(a)}
$$

From Eqs. 97 and 98, we obtain the relation

$$
\mathscr{E}-\mathscr{E}_{\min }=\sum_{a}\left(\mathrm{~A}_{a}-2 \mathrm{~A}_{a}^{\prime}\right) \overline{\mathrm{G}(\mathrm{t}) \mathrm{z}(\mathrm{t}) \Phi(a)}+\sum_{a} \mathrm{~A}_{a}^{\prime 2} \overline{\mathrm{G}(\mathrm{t}) \Phi(a)}
$$

Again, using Eq. 58, we can rewrite Eq. 99 as follows

$$
\mathscr{E}-\mathscr{E}_{\min }=\sum_{a}\left(\mathrm{~A}_{\alpha}^{2}-2 \mathrm{~A}_{a} \mathrm{~A}_{\alpha}^{\prime}+\mathrm{A}_{\alpha}^{\prime 2}\right) \overline{\mathrm{G(t) \Phi (a)}}
$$

which is equivalent to

$$
\mathscr{E}-\mathscr{E}_{\min }=\sum_{a}\left(A_{a}-A_{a}^{\prime}\right)^{2} \overline{G(t) \Phi(a)}
$$


This equation is the desired expression for the error that is introduced when the system coefficients are changed from their optimum values $A_{a}$ to the values $A_{a}^{\prime}$.

There are several interesting points to notice about Eq. 101 and the equations that lead to it. First, we notice that $\mathscr{E}-\mathscr{E}_{\min }$ is always positive, since $G(t)$ and $\Phi(a)$ are non-negative functions. Thus, Eq. 101 shows that the optimum coefficients (the $A_{a}$ ) determined by Eq. 58 actually render the error a minimum. Next, we notice that as a consequence of the time-domain orthogonality of the $\Phi(a)$, each cell contributes independently to the expression for the system error (Eq. 97). This very convenient property of the gate-function representation of a nonlinear system enables us to directly and independently relate changes in any system coefficient to changes in the error $\mathscr{E}$. From Eq. 101, we see that the increase in error caused by a change in the $a^{\text {th }}$ coefficient from its optimum value $A_{a}$ to $A_{a}^{\prime}$ is just

$$
\mathscr{E}-\mathscr{E}_{\min }=\left(\mathrm{A}_{a}-\mathrm{A}_{a}^{\prime}\right)^{2} \overline{\mathrm{G}(\mathrm{t}) \Phi(a)}
$$

But recall (Fig. 13) that $\overline{\mathrm{G}(\mathrm{t}) \Phi(\alpha)}$ is a quantity that we must evaluate in determining the optimum system. Hence, if any coefficient is changed from its optimum value (as it may be for purposes of approximating a system by a simpler system, as we shall see), we can immediately write the corresponding increase in error. Finally, we notice the interesting fact that for $G(t) \equiv 1$ (i.e., the mean-square-error criterion) the increase in error introduced by a given change in a coefficient is proportional to the probability that the corresponding cell in function space is occupied (recall that $\overline{\Phi(a)}$ is equal to the probability that the $a^{\text {th }}$ cell is occupied).

We now introduce another error criterion and relative to it we examine the effect of changing the system coefficients. For the criterion we specify an amplitude tolerance band $\mathrm{T}$ for a system output and regard two systems as approximately equivalent if their outputs (for any input that is common to both systems) coincide within this tolerance band. $T$ can be chosen to have a constant width for all amplitudes of the system output or its width can be chosen as a function of the amplitude of the system output (examples of these choices are illustrated later). Now recall that, in the gate-function representation of a nonlinear system, the output of the system at any instant is equal to the value of the coefficient $A_{a}$ that corresponds to the occupied cell in the function space. Hence, if we change the $a^{\text {th }}$ coefficient from $A_{a}$ to $A_{a}^{\prime}$, then the system output will change from $A_{a}$ to $A_{a}^{\prime}$ when the $a^{\text {th }}$ cell is occupied. At all other times (i.e., when the $a^{\text {th }}$ cell is not occupied) the system output will be the same as that before the change was made. Notice that this result holds regardless of the system input. As a consequence of this simple relation between changes in the coefficients and changes in the amplitude of the system output, we can directly transfer the tolerance band on the system output to the coefficients. That is, for example, if we choose a tolerance band 2 volts wide about the output waveform, then we can alter any and all of the coefficients by as much as \pm 1 volt in amplitude and the system output will remain within this tolerance band. 
In the optimum filter problem (for the case $G(t) \equiv 1$ ) if we alter any coefficient from its optimum value $A_{a}$ to $A_{a}^{\prime}$, in addition to knowing that the system output will change from $A_{a}$ to $A_{a}^{\prime}$ when the $a^{\text {th }}$ cell is occupied, we know the probability of the occurrence of this error. This probability is just $\overline{\Phi(a)}$ which was measured in the process of determining $\mathrm{A}_{a}$.

In summary, when any filter coefficient is changed from its optimum value $A_{a}$ to $A_{a}^{\prime}$, we can immediately determine the following quantities:

1. The change in the weighted mean square error.

2. The tolerance band relating the optimum and the altered systems.

3. For the case $G(t) \equiv 1$, the probability that the output of the altered system will differ from that of the original optimum system.

Conversely, if we specify upper limits on any of these quantities, we can find the permissible variation in the $A_{a}$. In this respect, it is most convenient to specify a tolerance band, for, as we have seen, the same tolerance band can be applied to the system coefficients, thus directly determining their maximum permissible variation. Furthermore, of the three quantities listed above, the tolerance band is the only one whose specification determines the permissible range of the $\mathrm{A}_{a}$ independently of the system input. Hence, this criterion is truly characteristic of the system itself. (More precisely, it is characteristic of the gate-function representation of the system.) Henceforth, when we speak of two systems as being nearly equivalent it is understood that this "equivalence" holds with respect to some suitably chosen tolerance band. To illustrate these concepts we consider an example of a nearly linear system.

EXAMPLE 7. Let the coefficients of an optimum mean-square filter be the same as those of example 6 with the one difference that $a_{222}=3.5$ instead of 3.0. The graphs of these coefficients are shown in Fig. 28. It is recognized that these graphs do not satisfy the conditions (sec. 4.1) for synthesis in the simple form of Fig. 23. However, let us establish a tolerance band of \pm 0.5 volt about the system output and ask if there is a linear system that is nearly equivalent to this system. In Fig. 28 the dashed lines in each graph indicate the \pm 0.5 -volt tolerance band. Inspection of this figure shows immediately that the system is nearly linear with respect to this tolerance band. All the graphs are linear except one (shown circled) and this one can be made linear, with the same slope as the other graphs of set 1, without exceeding the tolerance band, as shown by the dotted line. Hence this system is nearly equivalent to that of example 6 and can thus be approximated by the simple linear system of Fig. 27. Furthermore, by Eq. 102, the increase in the mean square error, when this optimum system is approximated by the linear system, is

$$
\mathscr{E}-\mathscr{E}_{\min }=(3.5-3.0)^{2} \overline{\Phi(2,2,2)}
$$

where $\overline{\Phi(2,2,2)}$ is one of the measurements that was made in determining $a_{222}$. Finally, we see that the output of the linear system of Fig. 27 will differ from that of the optimum 


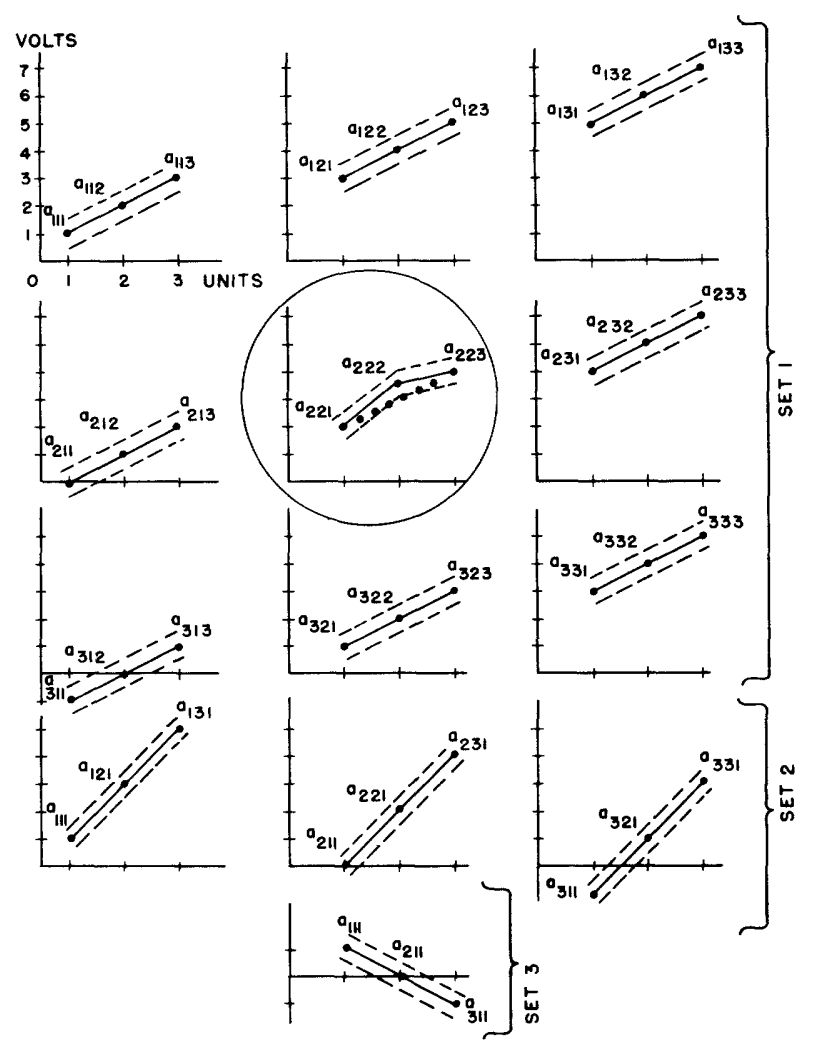

Fig. 28. Graphs of the coefficients of a nearly linear system.

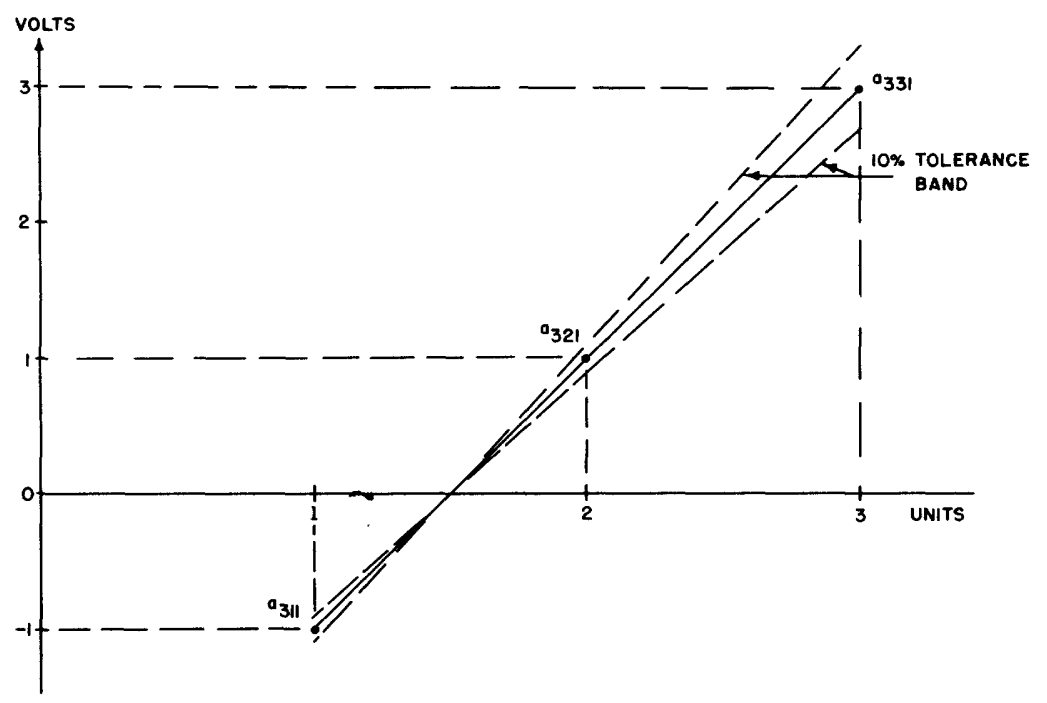

Fig. 29. Example of a 10 per cent tolerance band on a graph of system coefficients. 
system only when the 2,2,2 cell is occupied and then this output will be 3.0 volts instead of 3.5 volts. The probability of this error is the known quantity $\overline{\Phi(2,2,2)}$.

In this simple example only one graph is nonlinear. However, the general procedure for finding a linear or simple nonlinear system of the class shown in Fig. 23 (if it exists) to approximate any system is clear. We draw tolerance limits on the graphs of the coefficients and then see if we can fit into these limits curves that satisfy the conditions of the class of systems of Fig. 23.

In example 7 a tolerance band was chosen that had a constant width for all amplitudes of the signal output. In some applications it may be desirable to choose a band whose width is a function of the amplitude of the system output. For example, suppose we choose a band whose width is proportional to the system output. This corresponds to a percentage error criterion. That is, a system that approximates a given system with respect to this criterion has an output that is within a fixed percentage of the output of the given system at all times. An illustration of a 10 per cent tolerance band applied to a graph of the coefficients is shown in Fig. 29.

\section{NOTES ON THE APPLICATION OF THE THEORY}

\subsection{REDUCING THE COMPLEXITY OF SYSTEMS BY EXTRACTING SIMPLE SYSTEMS}

In section 4.3 we considered the approximation of nonlinear filters by linear and simple nonlinear filters. The object of this approximation is to reduce the complexity of the filter. In many cases we may find that, for a given tolerance band, there exists no system of the form shown in Fig. 23 that approximates a given system. It may, however, still be possible to simplify the synthesis of this system by dividing it into two or more parts, each of which can be synthesized in a relatively simple form. The basis for the division of the system into separate parts is provided by the gate-function representation of nonlinear systems that is given in Eq. 53. This representation lends itself conveniently to the decomposition of systems into parallel connected component systems. For any cell of the given system, the coefficient $A_{a}$ is just the sum of the coefficients of the corresponding cell in each of the parallel connected component systems. Hence, we can extract a simple system from a given system by subtracting the coefficients of the simple system from the corresponding coefficients of the given system. If the extraction simplifies the original system, then we have succeeded in breaking one complex system into two less complex parts. To illustrate this, let us consider example 7. Instead of approximating the system of this example by a linear one, let us synthesize it as the parallel combination of two simple systems. We note (Fig. 28) that, except for the coefficient $a_{2,2,2}$, all the graphs of the coefficients satisfy the conditions for a linear system. Let us subtract the set of coefficients corresponding to this linear system from the coefficients given in Fig. 28. The result is that all the coefficients of the remaining system are zero except the one corresponding to the $2,2,2$ cell, which is 
0.5. The coefficients of the extracted linear system are those of example 6. Hence, the nonlinear system of example 7 can be synthesized as the parallel combination of the linear system of Fig. 27 and a very simple nonlinear system that has only one coefficient, $a_{2,2,2}=0.5$. This approach of extracting simple systems to reduce the complexity of a system is effective only when, as in this example, the extraction causes many of the coefficients of the remaining system to be zero, thereby simplifying its synthesis.

\subsection{OPTIMIZATION OF THE LAGUERRE FUNCTION SCALE FACTOR}

It has been assumed throughout that the scale factor associated with the argument of the Laguerre functions is unity. By substituting $\mathrm{kx}$ for $\mathrm{x}$ in Eq. 1 we obtain the Laguerre functions that have the scale factor $k$. But we have no convenient analytical method for determining the optimum value of $\mathrm{k}$ in the filter problem. Recall that the impulse response at the $n^{\text {th }}$-output terminal of the Laguerre network is $h_{n}(t)$ or $h_{n}(k t)$ with the scale factor $k$. We see that $k$ effects a time scaling of the impulse response of the Laguerre network and hence a frequency scaling of the transfer function of this network. In determining optimum filters by the methods that have been described we can make use of our knowledge of the frequency band of $x(t)$ to judiciously choose $k$. It would be convenient, however, to have a method for obtaining the optimum $\mathrm{k}$ rather than just a good guess for it. An experimental procedure for obtaining the optimum $\mathrm{k}$ will now be described.

Consider the effect of changing the time scale of $x(t)$ and $z(t)$ in the experimental procedure for determining optimum filters. That is, consider that these time functions are replaced by $x(t / k)$ and $z(t / k)$. In the laboratory this could be achieved by recording the ensemble members of $x(t)$ and $z(t)$ and then playing the recording back at a speed of $1 / \mathrm{k}$ times the recording speed. First, consider the effect of this time scaling on the determination of no-storage filters in accordance with Fig. 9. It is clear that, since the apparatus for determining the filters has no memory, its operation is independent of the time scaling. That is, the same filter will be determined regardless of the value of k. Now consider the procedure (Fig. 13) for determining optimum filters involving memory. We notice that the only portion of the apparatus that involves memory is the Laguerre network. As a consequence, if we replace $x(t)$ and $z(t)$ by $x(t / k)$ and $z(t / k)$, we obtain the same filter as if we had used $x(t)$ and $z(t)$ but changed the scale factor of the Laguerre network from unity to $k$. Now recall (sec. 3.6) that apparatus can be constructed that indicates the minimum error for the optimum filter (of the class of filters with a given Laguerre network and $\Phi$ generator). This apparatus, like that shown in Fig. 13, consists of no-memory circuits and a Laguerre network. By varying the speed of the recordings of $x(t)$ and $z(t)$ which are fed into this apparatus and observing its output ( $\mathscr{E}_{\mathrm{min}}$ of Eq. 62 ) we can directly determine the optimum k. In practice, we would build the Laguerre network in the minimum error detecting apparatus so that it would have a scale factor corresponding to our judicious guess of the optimum $\mathrm{k}$. Then we could vary the speeds of the recordings of $x(t)$ and $z(t)$ in order to explore scale 
factors in the neighborhood of our guess. We should note that, in general, the amplitude of the signals at the outputs of the Laguerre network will change with the change in speed of the recordings of $x(t)$. The maximum amplitude of these signals must be kept at a constant level that corresponds to the range $a-b$ (Eq. 37) over which the gate functions are defined. If desired, circuitry can be constructed to accomplish this adjustment automatically.

\subsection{CHOOSING THE FUNCTIONS THAT CHARACTERIZE THE PAST OF THE FILTER INPUT}

For the theoretical discussions of the preceding sections it was convenient to characterize the past of the filter input by Laguerre functions, since they form a complete set of functions on this past. In practice, however, we shall only use a small number of Laguerre functions. Hence, the fact that they form a complete set is no longer of prime importance to us. The important question in the practical case is, "How well can we do with a small number of functions of the past?"

In many filter problems involving the separation of signals from noise the immediate past of the filter input contains more information about the present value of the desired signal than does the more remote past. Since the Laguerre functions decay exponentially (Eq. 1), they weight the immediate past of the input considerably more than the more remote past and, therefore, we expect that they will form a convenient set of functions for use in many filter problems.

For some applications, it may be convenient to derive the functions of the past from taps on a delay line rather than from a Laguerre network (even though the outputs of a delay line do not form a complete set of functions on the past of the input). For example, suppose we are dealing with messages in which consecutive symbols are coded in a sequence of pulses of different amplitudes. Then it would be appropriate to replace the Laguerre network by a delay line with taps so spaced that consecutive pulses appear at adjacent taps. Then at any instant the s-outputs of the delay line would specify the amplitudes of the s preceding pulses. Another example, for which it is appropriate to replace the Laguerre network by a delay line, is the problem of detecting the presence of a train of pulses that are equally spaced in time. If the delay between the taps is equal to the time interval between the pulses, then, when the train of pulses is present, it will affect all outputs of the delay line simultaneously. These are examples in which it is desirable to give equal weight to different portions of a finite interval of the past, and thus a delay line is more appropriate than a Laguerre network.

It is clear that there are many choices that we can make for the functions that charac terize the past of the filter input. Each choice implies a restriction to a class of filters from which the experimental procedure, similar to that indicated in Fig. 13, will pick the optimum for the particular problem. We can make use of the apparatus that determines the minimum error for a given class of filters (mentioned in secs. 3.6 and 5.2) in order to decide whether to use a Laguerre network or a delay line, and so forth, in 
a given problem. We insert the different networks into this apparatus in place of the Laguerre network and choose the one that yields the smallest error.

\subsection{CHOOSING THE NUMBER OF GATE FUNCTIONS}

By increasing the number of gate functions that are associated with each Laguerre coefficient we quantize the function space of the input into smaller cells. While, in general, this is desirable from the point of view of reducing the filtering error, it leads to a very large number of coefficients that have to be evaluated. In applying the theory we should, when possible, make use of any information about the particular filter problem that will enable us to reduce the number of gate functions. For example, consider the problem of pure (noiseless) prediction of a stationary time function consisting of equally spaced pulses with amplitudes of zero or unity with a certain probability distribution. As we found in the previous section, it is appropriate, for a problem of this kind, to replace the Laguerre network by a delay line. The output from each tap on the delay line will then take on only the values zero and unity. Hence, we need only two gate functions for each output of the delay line.

In the previous section we also mentioned that in many filter problems the immediate past of the input contains more information about the present value of the desired output than does the more remote past. A study of the Laguerre functions shows that the higherorder functions weight the remote past more strongly than do the lower-order functions. Hence, we expect that, for purposes of filtering, it might not be as important to distinguish small changes in the amplitude of the higher-order Laguerre coefficients as in the lower-order ones (or, in the case of delay lines, it might not be as important to distinguish small changes in those outputs that represent samples of the more remote past). Thus we might choose a smaller number of gate functions for the higher-order Laguerre coefficients than for the lower-order ones.

In summary, the choices of the scale factor, the functions that characterize the past of the input, and the number of gate functions are all choices that determine the class of filters from which the procedure, similar to that indicated in Fig. 13, determines the optimum filter. We should use any information about the particular filter problem that will enable us to judiciously choose the class of filters so that the number of coefficients is reasonable in the light of the task that the filter is to perform.

\section{Acknowledgment}

The author wishes to express his deep appreciation to Professor Y. W. Lee, who first suggested a study of the Wiener theory of nonlinear system characterization and supervised the research that resulted from this study. The constructive criticism of Professor Lee in the research and in the writing of this report led the work to its present form. The author is also indebted to Professor Norbert Wiener, who has been a source of inspiration through his explanations and discussions of his theory. 


\section{References}

1. N. Wiener, Mathematical problems of communication theory, Summer Session Lecture Notes, 1953-1954, M.I.T. (unpublished).

2. Y. W. Lee, Synthesis of electric networks by means of the Fourier transforms of Laguerre's functions, J. Math. Phys. 2, 83-113 (June 1932).

3. E. C. Titchmarsh, Introduction to the Theory of Fourier Integrals (Oxford University Press, London, 1937), pp. 76-82.

4. N. Wiener, The Fourier Integral and Certain of its Applications (Dover Publications, Inc., New York, 1933).

5. H. E. Singleton, Theory of nonlinear transducers, Technical Report 160, Research Laboratory of Electronics, M.I.T., Aug. 12, 1950, pp. 30-32.

6. Y. W. Lee, Statistical theory of communication, Course 6.563 Class Notes, 1952, M.I.T. (unpublished).

7. N. Wiener, Extrapolation, Interpolation, and Smoothing of Stationary Time Series (John Wiley and Sons, Inc., New York, 1949).

8. L. A. Zadeh, Optimum nonlinear filters for the extraction and detection of signals, a paper presented at the IRE National Convention, New York, March 25, 1953.

9. T. E. Stern, Piecewise-linear analysis and synthesis, a paper presented at the Symposium on Nonlinear Circuit Analysis II, Polytechnic Institute of Brooklyn, April 26, 1956. 